\title{
Model of the early development of thalamo-cortical connections and area patterning via signaling molecules
}

\author{
Jan Karbowski ${ }^{1, *}$ and G.B. Ermentrout ${ }^{2}$ \\ ${ }^{1}$ Sloan-Swartz Center for Theoretical Neurobiology, Division of Biology 216-76, \\ California Institute of Technology, Pasadena, CA 91125, USA \\ ${ }^{2}$ Department of Mathematics, University of Pittsburgh, Pittsburgh, PA 15260, USA
}

\begin{abstract}
The mammalian cortex is divided into architectonic and functionally distinct areas. There is growing experimental evidence that their emergence and development is controlled by both epigenetic and genetic factors. The latter were recently implicated as dominating the early cortical area specification. In this paper, we present a theoretical model that explicitly considers the genetic factors and that is able to explain several sets of experiments on cortical area regulation involving transcription factors Emx2 and Pax6, and fibroblast growth factor FGF8. The model consists of the dynamics of thalamo-cortical connections modulated by signaling molecules that are regulated genetically, and by axonal competition for neocortical space. The model can make predictions and provides a basic mathematical framework for the early development of the thalamo-cortical connections and area patterning that can be further refined as more experimental facts become known.
\end{abstract}

Keywords: cerebral cortex, development, thalamo-cortical connections, cortical area patterning, signaling molecules.

Running Title: cortical patterning via signaling molecules

* Corresponding author at:

Email: jkarb@cns.caltech.edu

Phone: (626)-395-5840

Fax: (626)-795-2397 


\section{Introduction}

Neocortex is organized into many functional subdivisions called areas that have sharp boundaries. The areas can be identified anatomically by investigating their distinct cytoarchitectonic properties and unique connectivity patterns. One interesting feature is that the neocortical map composed of these areas is highly conserved within the same species, and has common properties across different species with different brain sizes (Nauta and Feirtag, 1986; Hofman, 1989; Finley, 1995; Northcutt and Kaas, 1995; Krubitzer, 1995; Karbowski, 2003).

One of the main questions in the development of the mammalian cortex is what factors control the specification and differentiation of cortical areas. In the past, there were two opposing views. One proposition was that areas are specified by intrinsic genetic factors (Rakic, 1988) - the so-called protomap model. Another proposition was that areas are specified by extrinsic influence, i.e. by thalamo-cortical inputs (O'Leary, 1989). In recent years, however, the consensus has been growing that both of these factors, i.e. genetic and epigenetic contribute to the cortical area patterning (Krubitzer and Huffman, 2000; O'Leary and Nakagawa, 2002). The genetic effects are thought to dominate the early stages of the development, while the epigenetic effects influence the later stages. This view has been stimulated by recent experiments demonstrating a direct genetic involvement in cortical arealization (Bishop et al, 2000; Mallamaci et al, 2000; Fukuchi-Shimogori and Grove, 2001, 2003; Bishop et al, 2002; Muzio et al, 2002; Garel et al, 2003). In particular, it has been found that the genetic transcription factors Emx2 and Pax6 control area specification even before thalamo-cortical input 
arrives (Bishop et al 2000; Mallamaci et al 2000; Muzio et al, 2002; Bishop et al, 2002). Both of these factors are expressed in a graded and complementary manner along antero-posterior $(\mathrm{A} / \mathrm{P})$ axis in the neocortical ventricular zone. In mice with Emx2 mutation, anterior areas expand and posterior areas shrink, in Pax6 mutants the opposite is observed. Another set of experiments has found that cortical maps can be disrupted by modifying fibroblast growth factor FGF8 (Fukuchi-Shimogori and Grove, 2001, 2003; Garel et al, 2003), which is also involved in embryonic patterning. In wild type mouse neocortex, the FGF8 source is located in the anterior pole giving rise to an expression concentration decaying towards the posterior end. Under such regular conditions, the barrel field ( $\mathrm{S} 1$ area) is positioned in the center along A/P axis. However, increase in the expression of FGF8 at the anterior pole, displaces the barrel field more posteriorly. In contrast, blocking the FGF8 activity with a soluble FGF8 receptor moves the field in the opposite direction. Moreover, introduction of an extra source of FGF8 at the posterior pole creates an additional barrel field that partly duplicates the original field. Taken together, all these results suggest that genetic perturbations can have a profound effect on the development of thalamo-cortical connections and area patterning. Additionally, these results are consistent with a hypothesis that the above signaling molecules provide positional information for neuroepithelial cells (Wolpert, 1969, 1996; Grove and Fukuchi-Shimogori, 2003).

Area-specific TC projections are probably controlled by axon guidance molecules, similar to what happens in the retino-tectal system (O'Leary et al, 1999). Although several molecules have been found that are able to guide TC axons, there are some 
experimental indications that the plausible candidates for the late phase of accurate TC targeting are ephrins and their receptors that are expressed both in the neocortex and in the thalamus (Vanderhaeghen et al, 2000; Mackarehtschian et al, 1999; Mann et al, 2002; Uziel et al, 2002; Takemoto, 2002). It is well established that these molecules direct axons to appropriate locations in many systems, in particular, in the retinotectal system (Goodhill and Richards, 1999), which, like the neocortex, preserves the topography of projections.

The relationship between the axon guidance molecules and Emx2, Pax6, and FGF8 is not known at present. It is likely, however, that the latter control the expression level and gradients of the guiding molecules. Thus, if the transcription factors and FGF8 concentrations are modulated, then it should affect the concentration pattern of the axon guidance molecules. This hypothesis is consistent with the experiments on cortical areas shifting described above.

The purpose of this article is to provide a theoretical model of the early TC projections and cortical area patterning. The key assumption we make is that the signaling molecules Emx2, Pax6, and FGF8 control the axon guidance molecules, which in turn control TC pathfinding. We assume that there are three main types of the axon guidance molecules, which we call A, B and C, that are expressed in the neocortical ventricular zone in a graded and complementary manner along $\mathrm{A} / \mathrm{P}$ axis. In the model, these molecules repel and attract different TC axons, which branch diffusively along the cortex, with specific forces, different for different classes of axons. Additionally, axons interact among themselves by competing for neocortical space. As a consequence of 
these interactions, a pattern of TC connectivity emerges that divides the neocortex into regions with sharp boundaries defined by distinct TC axon types. In this framework, area shifting experiments described above can be understood as a result of shifts in the patterns of expression of the signaling molecules.

\section{Model}

We assume that the early $\mathrm{TC}$ connectivity and cortical regionalization along $\mathrm{A} / \mathrm{P}$ axis is established by chemo-interaction between axons originating from the thalamus and axon guidance molecules located on the cortex (Sperry, 1963). In this paper, we assume that there are $N=5$ main axon types. Under normal conditions, type $i=1$ corresponds to the axons coming from ventrolateral thalamus and terminating in primary motor area M1, type $i=3$ corresponds to the axons originating from thalamic ventrobasal complex and terminating in $\mathrm{S} 1$, type $i=5$ relates to the axons connecting thalamic LGN with the primary visual area V1. The remaining axons of type 2 and 4 terminate between areas M1 and S1 (type $i=2$ ), and between S1 and V1 (type $i=4$ ), in order to ensure a topographic TC connectivity.

In the model, it is assumed that there are three types of the axon guidance molecules A, B, and C. In our choice of the number of guiding molecules, we were motivated by experimental indications that the best candidates for the late phase of precise TC pathfinding are ephrins and their receptors (O'Leary and Nakagawa, 2002; Lopez-Bendito and Molnar, 2003). Up to now, 3 types of ephrins have been implicated as important: ephrin-A5 expressed at high levels in the central part of the cortex, ephrin-A4 ex- 
pressed in the cortical intermediate zone with a gradient decaying from the anterior towards the posterior end, and ephrin-B3 with a complementary gradient to ephrin-A4 (Lopez-Bendito and Molnar, 2003). In the model, the molecule A may mimic the action of ephrin-A4, the molecule B may act analogously to ephrin-A5, and the molecule C may mimic ephrin-B3. The molecules A, B, and $\mathrm{C}$ have a mixed effect on TC axons, i.e. they both repel and attract them and this is encoded in the interaction matrix $\gamma$. Experimentally, ephrin-As and -Bs ligands have been shown to exhibit some selective behavior, too. Ephrin-B expressing cells tend to attract axonal growth cones with EphB receptors (McLaughlin et al, 2003), and repulse axons with EphA4 receptors (Takemoto et al, 2002). The prevailing action of ephrin-As expressing cells is repulsion, which is well documented with axons expressing EphA (e.g., Mann et al, 2002; McLaughlin et al, 2003). However, when axonal cones express both EphA and ephrin-A as receptors, then cells with ephrin-As can attract them (Knoll and Dreschner, 2002).

In addition to the chemo-affinity, we assume that axons branch randomly along $\mathrm{A} / \mathrm{P}$ axis. Axonal branching has been found in the retino-tectal (Yates et al, 2001) and retinocollicular (Simon and O'Leary, 1992) systems, and there are some indications (Mann et al, 2002) that similar effects are present in the establishment of TC connectivity. The basics of the process of random growth and decay of branches along axonal length can be mathematically described by some stochastic component acting on a local branching density (see below). The combined effect of the chemo-interactions and stochasticity on axonal terminals is mathematically equivalent to their biased random growth on the cortical surface. The bias in growth is caused by the gradients of the guidance 
molecules. For attractive interactions, the larger the gradient the larger the growth rate. For repulsive interactions, the larger the gradient the larger the decay rate.

Another crucial assumption is that there exists some sort of repulsive interaction between TC axons of different types that leads to their competition for neocortical space. This constraint implies that the density of TC projections is limited, because not all axon types are allowed to terminate simultaneously at a single point in the cortex. The idea of axon-axon competition was used in the past in the context of retino-tectal mapping (Prestige and Willshaw, 1975; Fraser and Hunt, 1980) and it is consistent with recent experiments in retino-collicular system (Feldheim et al, 2000).

In the mathematical model, the development of TC connections $\left(c_{i}\right)$ between axons of type $i$ (with the branching density $a_{i}$ ) and cortical neurons $n$ is represented by a simple kinetic reaction scheme: $n+a_{i} \rightleftarrows_{\alpha_{i}}^{\beta_{i}} c_{i}$ (e.g. Murray, 1993). Connections are destroyed with the rate $\alpha_{i}$, and created with a rate proportional to the product of the fraction of available neurons $n(x)$ and some power of branching density, i.e. $\beta_{i} n(x)\left[a_{i}(x)\right]^{k}$, where $\beta_{i}$ is some positive constant. When the exponent $k>1$, then TC connections are created with a higher rate in regions with high concentration of axonal branches, so that there is some cooperativity. (In our numerical simulations we take $k=3$.)

The full set of differential equations describing the above processes is given by:

$$
\begin{gathered}
\frac{\partial c_{i}(x)}{\partial t}=-\alpha_{i} c_{i}(x)+\beta_{i} n(x)\left[a_{i}(x)\right]^{k}, \\
\frac{\partial a_{i}(x)}{\partial t}=\frac{\partial J_{i}(x)}{\partial x}+\alpha_{i} c_{i}(x)-\beta_{i} n(x)\left[a_{i}(x)\right]^{k},
\end{gathered}
$$




$$
n(x, t)+\sum_{i=1}^{N} c_{i}(x, t)=1
$$

with the flux current

$$
J_{i}(x)=D \frac{\partial a_{i}}{\partial x}-a_{i}(x)\left(\gamma_{A i} \frac{\partial \rho_{A}}{\partial x}+\gamma_{B i} \frac{\partial \rho_{B}}{\partial x}+\gamma_{C i} \frac{\partial \rho_{C}}{\partial x}\right)
$$

where $c_{i}(x)$ is a fraction of TC connections of type $i$ at position $x$ on the developing cortex, $n(x)$ is a fraction of cortical neurons at point $x$ available for a TC connection (i.e. not already connected by other TC axons), $a_{i}(x)$ is the branching density of axons of type $i$ at point $x$ on the cortex that originate from the thalamus, and $N$ is the number of axon types. Our model is a population model, which deals with densities and fractions, and does not take directly into account the fine structure of axonal branching on a single axon level, such as complex arborization patterns; instead we consider population branching density. (For detailed modeling of branching structures and their influence on the retino-topic map, see Yates et al, 2004). Eq. (3) is a mathematical consequence of axonal competition for neocortical space, and it provides a conservation law for the fractions of available (unconnected) neurons and all the connection types for every point on the cortex and for all times. The flux $J_{i}(x)$ is associated with the growth and decay of axonal branches of type $i$ at point $x$. It is composed of the following contributions: the diffusive axonal branching that is proportional to the diffusion constant $D$, and chemo-interaction between axonal branches and the axon guidance molecules that have concentrations $\rho_{A}(x), \rho_{B}(x)$, and $\rho_{C}(x)$ (Sperry, 1963). The nature of this interaction 
is additive, that is, the guiding molecules do not interact with themselves, and it can be either repulsive or attractive, which is controlled by the sign and strength of the parameters $\gamma_{A i}, \gamma_{B i}$, and $\gamma_{C i}$.

Our model focuses on how the anterior/posterior boundaries of cortical areas are formed. Thus, from a theoretical point of view, this biological problem has a onedimensional character and the spatial variable $x$ measures distances along the $\mathrm{A} / \mathrm{P}$ axis. In our model, the anterior pole corresponds to $x \approx 0$, and the posterior pole to $x \approx L$, where $L$ is the linear size of the cortex in that direction.

We adopt sealed-end boundary conditions for the flux current $J_{i}(x)$, i.e. $J_{i}(L)=$ $J_{i}(0)=0$, since in our system axonal branches cannot grow outside the boundaries of the cortex. This choice implies that the total number of axonal branches and connections of any given type in the system is constant, i.e. $\int_{0}^{L} d x\left[a_{i}(x)+c_{i}(x)\right]$ is time independent (this follows from adding eqs. (1) and (2)). Thus initially, there are many branches but no connections. However, as time progresses, the total number of branches decreases at the expense of the formed connections. In a real biological system, however, this constraint may be satisfied only approximately.

It is assumed that the axon guidance molecules located on the cortex are regulated by the transcription factors Emx2 and Pax6, and the fibroblast growth factor FGF8. Recent experiments (Fukuchi and Grove 2003; Garel et al, 2003) show that (i) FGF8 and Emx2 mutually inhibit each other. The Fukuchi and Grove (2003) results also suggest that (ii) FGF8 directly controls the location of cortical maps, and additionally that (iii) Emx2 indirectly controls the positioning of cortical maps by acting upstream 
of FGF8 and regulating it. Another set of experiments (Muzio et al, 2002) shows that (iv) the two transcription factors, Emx2 and Pax6, mutually repress each other. Based on these facts, one can construct a minimal signaling pathway (Fig. 1) that is responsible for regulating expression levels of the guiding molecules A, B, and C. In this model, both Emx2 and Pax6 act upstream of FGF8, but only Emx2 regulates it directly. FGF8 and Pax6 have similar gradients, i.e. their concentrations decay from the anterior towards the posterior end, while Emx2 has an opposite gradient to them. The output signal $f$ from the pathway can be viewed as a renormalized (by interactions with other signaling molecules) concentration of FGF8. We assume that either this signal or some other $f$-activated signal serves as a morphogen signal (Grove and Fukuchi-Shimogori, 2003) that provides a positional information for cortical cells, similar to what happens in other developing systems (Wolpert, 1969, 1996). In response to the signal $f$, cells express different levels of the guiding molecules, which in turn control TC axons.

The diagram in Fig. 1 can be described mathematically in the following way. The transcription factors Emx2 and Pax6 both repress each other with strengths $v_{1}$ and $v_{2}$, respectively $\left(v_{1}, v_{2}>0\right)$. Emx2 negatively controls FGF8 with a strength $w_{1}$, and FGF8 in turn inhibits Emx2 with a strength $w_{2}\left(w_{1}, w_{2}>0\right)$. From all of these relationships, it follows that FGF8 $(f)$ is regulated negatively by Emx2 and positively by Pax6. Mathematical equations describing the dynamics of the expression of Emx2, Pax6, and FGF8 are given by

$$
\tau_{s} \frac{d s(x)}{d t}=-s(x)+\frac{\eta_{e m x}(x)}{1+w_{2} f(x)+v_{2} r(x)}
$$




$$
\begin{gathered}
\tau_{r} \frac{d r(x)}{d t}=-r(x)+\frac{\eta_{p a x}(x)}{1+v_{1} s(x)} \\
\tau_{f} \frac{d f(x)}{d t}=-f(x)+\frac{\eta_{f g f}(x)}{1+w_{1} s(x)}
\end{gathered}
$$

where $s(x), r(x)$, and $f(x)$ are expression levels (renormalized concentrations) of Emx2, Pax6, and FGF8, respectively, in the presence of interactions between these molecules. The parameters $\tau_{s}, \tau_{r}$, and $\tau_{f}$ are signaling time constants, and finally $\eta_{e m x}(x), \eta_{f g f}(x)$, $\eta_{\text {pax }}(x)$ are uncoupled (i.e. without interactions) concentrations of Emx2, FGF8, and Pax6. We assume the following forms of the uncoupled concentrations for Emx2, Pax6, and FGF8: $\eta_{e m x}(x)=A_{e m x} \exp \left[-(x-L)^{2} / \zeta_{e m x}^{2}\right], \eta_{p a x}(x)=A_{\text {pax }} \exp \left[-x^{2} / \zeta_{\text {pax }}^{2}\right]$, and $\eta_{f g f}(x)=A_{f g f} \exp \left[-x^{2} / \zeta_{f g f}^{2}\right]$, where $A_{e m x}, A_{\text {pax }}, A_{f g f}$ are amplitudes of expression, and $\zeta_{e m x}, \zeta_{p a x}, \zeta_{f g f}$ are constants characterizing the ranges of expression with values in the interval $(0, L)$. When two sources of FGF8 are present, one in the anterior and second in the posterior end, then the uncoupled concentration of FGF8 takes the form: $\eta_{f g f}(x)=A_{f g f} \exp \left[-x^{2} / \zeta_{f g f}^{2}\right]+A_{f g f}^{\prime} \exp \left[-(x-L)^{2} / \zeta_{f g f}^{\prime 2}\right]$, where $A_{f g f}^{\prime}$ and $\zeta_{f g f}^{\prime}$ are the amplitude and the range of the posterior source, respectively. These uncoupled concentrations act as signals produced at boxes denoted by Emx, FGF, and Pax in Fig. 1. We assume that the time constants $\tau_{s}, \tau_{r}$, and $\tau_{f}$ are much smaller than characteristic time constants associated with the processes involving TC projections. That is, at the time when TC axons arrive to the cortical surface, all these molecules have already reached their steady-state, which is consistent with experimental data (Cohen-Tannoudji et al, 1994; Miyashita-Lin et al, 1999; Nakagawa et al, 1999). For this reason, we are interested in a steady-state solution for $f(x)$. Its plot is displayed in 
Fig. 2a. Under normal conditions, its spatial profile displays a monotonic decay from the anterior towards the posterior end (Fig. 2a), i.e. it has a similar gradient to the uncoupled FGF8.

We assume that the stationary distributions of the guiding molecules are as follows: the molecule $\mathrm{A}$ is mostly expressed in those regions of the cortex for which the signal $f(x)$ is high (anterior end), B is expressed mostly in regions where $f(x)$ is moderate (center), and $\mathrm{C}$ has high concentration in locations where $f(x)$ is weak (posterior end). These relationships can be represented mathematically in the following way:

$$
\begin{gathered}
\rho_{A}(x)=G_{A}\left(f(x)-\theta_{1}\right), \\
\rho_{B}(x)=G_{B}\left(\theta_{2}-f(x)\right) G_{B}\left(f(x)-\theta_{3}\right), \\
\rho_{C}(x)=G_{C}\left(\theta_{4}-f(x)\right),
\end{gathered}
$$

where $\rho_{A}, \rho_{B}$, and $\rho_{A}$ are stationary concentrations of the guiding molecules $\mathrm{A}, \mathrm{B}$, and C. The thresholds $\theta_{i}(i=1, \ldots, 4)$ are some positive constants such that $\theta_{4}<\theta_{3}<\theta_{2}<$ $\theta_{1}<f(0)$, and $G_{j}(y)=\left(\kappa_{j} / 2\right)\left[1+\tanh \left(y / \sigma_{j}\right)\right]$, where $\kappa_{j}$ and $\sigma_{j}$ (for $j=A, B, C$ ) are some positive constants controlling the amplitude and the slope of the concentration $\rho_{j}(x)$. The parameters $\sigma_{j}$ are chosen such as to ensure that the expressed levels of the guiding molecules A, B, and C have graded concentrations (Fig. 2b). 


\section{Results}

All the results reported in this section were obtained by numerically solving eqs. (1)(10). We used a second-order Runge-Kutta method for the ordinary differential equations and a Crank-Nicholson method (second order accurate in time and space) for the partial differential equations. The values of the parameters used are given in the legend of Fig. 2.

\section{Normal area positioning.}

Under normal conditions (wild type) cortical areas in the mouse neocortex are located such that motor area M1 occupies anterior part, sensory area S1 occupies the central part, and visual area V1 is positioned in the posterior end. Areas are defined as a spatial pattern of TC connectivity fraction $c_{i}(x)$ for $i=1, \ldots, 5$. A high value of $c_{1}(x)$ corresponds to the M1 field, a high value of $c_{3}(x)$ corresponds to the S1 area, and a high value of $c_{5}(x)$ corresponds to V1. For the remaining two connection types $c_{2}(x)$ and $c_{4}(x)$, their high values correspond to areas between M1 and S1, and between S1 and $\mathrm{V} 1$, respectively. Both, the connectivity fraction $c_{i}(x, t)$ and the axonal branching density $a_{i}(x, t)$ evolve in time to a steady-state according to eqs. (1)-(4) (see Figs. 3 and 4). Initial conditions are chosen such that $a_{i}(x, t=0)$ is uniformly (although with some noise) distributed in space, and $c_{i}(x, t=0)=0$, for $i=1, \ldots, 5$. This choice is motivated by experimental data (Simon and O'Leary, 1992; Yates et al, 2001), suggesting that the early TC connectivity in mammals is established in three phases. The first phase is axonal overshooting along $\mathrm{A} / \mathrm{P}$ axis beneath the cortical plate. The second phase is composed of axonal branching along their length, and the final phase provides stabiliza- 
tion of topographically correct axonal collaterals and elimination of distant branches. Our modeling starts from the second phase. As time progresses both of the above distributions evolve into spatially heterogeneous state with a close relationship between patterns of $a_{i}(x)$ and $c_{i}(x)$ (compare Figs. 3c and $4 \mathrm{c}$ ). The reason for this relationship can be disclosed if we use eqs. (1) and (3). Then one can derive a formula relating the stationary distribution of the connections $\overline{c_{i}}(x)$ with the stationary branching densities $\overline{a_{i}}(x):$

$$
\overline{c_{i}}(x)=\frac{\beta_{i}\left[\overline{a_{i}}(x)\right]^{k}}{\alpha_{i}+\sum_{j=1}^{N} \beta_{j}\left[\overline{a_{j}}(x)\right]^{k}} .
$$

From this equation, it follows that the area of type $i$ emerges in regions with high concentration of the branches of type $i$. However, one should note that not all diffusive branches form connections. This can be seen in Fig. 3c, where branches of type 2 and 4 have rather broad distributions, which are absent in the connectivity pattern in Fig. 4c.

The locations of cortical regions are shaped by different forces coming from the axon guidance molecules A, B and C. These forces activate the growth of axonal branches in some locations and inhibit their growth in other locations. To have, at a steadystate, a high density of axons of type $i=1$ in the anterior end, it is assumed that they are moderately/strongly attracted by the molecules A, repulsed strongly by the molecules C, and repulsed moderately by B. Similarly, to have high density of axons of type $i=5$ in the posterior end, it is assumed that they are moderately/strongly 
attracted by $\mathrm{C}$, strongly repulsed by the molecules $\mathrm{A}$, and moderately repulsed by B. Axons of type $i=3$ are forced to aggregate in the center by assuming that they are attracted by B, and repulsed, either strongly or moderately, with approximately the same strength from the guiding molecules A and C. Elements of the interaction matrix $\gamma_{\alpha i}$ for axons of type $i=2$ and $i=4$ are all negative (repulsion), and are chosen such that these axons terminate in between axons 1, 3 and 3, 5 in the wild type case. Although the concentrations of A, B, and C are graded (Fig. 2b), the spatial pattern of the fractions of TC connections $c_{i}(x)$ is almost exclusive with sharp borders (Fig. 4c), which leads to the emergence of cortical areas (Fig. 4d). The exclusiveness comes from the axonal competition constraint we imposed (see eq. (3)). The width of the areal border is directly proportional to the diffusion constant $D$, and inversely proportional to the product of the coefficient $k$ and the magnitude of the interaction between guiding molecules and axons. Purely repulsive interactions increase the border width in comparison to cases with mixed repulsive/attractive interactions (see Fig. 9 in the discussion).

\section{Area shifting.}

In the area shifting experiments expression levels of Emx2, Pax6, and FGF8 were affected (Bishop et al, 2000; Mallamaci et al, 2000; Fukuchi-Shimogori and Grove, 2001; Bishop et al, 2002; Muzio et al 2002; Garel et al 2003, Fukuchi-Shimogori and Grove, 2003). We model this effect by reducing or amplifying the appropriate concentration

amplitude $\left(A_{e m x}, A_{\text {pax }}, A_{f g f}\right)$ and the range of expression of one of these molecules. In Fig. 5, we present posteriorly shifting areas as we decrease the amplitude $A_{\text {emx }}$ to zero. 
This case corresponds to loss-of-function Emx2 mutants for which such shifting has been reported (Bishop et al, 2000; Mallamaci et al, 2000; Muzio et al, 2002; Bishop et al, 2002). The interpretation of this effect in our model is as follows. Reducing the concentration of Emx2 effectively increases the output signal $f$ (see, Fig. 1), since then the Emx2 $\rightarrow$ FGF8 inhibition decreases. This leads effectively to shifting posteriorly the expression profile of $f$ (Fig. 5a), and consequently shifting posteriorly the expression pattern of the guiding molecules (Fig. 5b). That new pattern biases axonal branching growth to the right to new locations, where balanced forces act on them (Fig. 5c). Again, there is a close relationship between distributions of axons and TC connectivity patterns (Figs. 5c,d). All areas are shifted posteriorly (Fig. 5e). In particular, a clear shift in the position of the S1 area can be seen by comparing figures $4 \mathrm{c}$ and $5 \mathrm{~d}$.

Fig. 6 displays anteriorly shifting areas as we decrease the amplitude $A_{p a x}$ to zero. This case corresponds to loss-of-function Pax6 mutants for which shifting in this direction has been experimentally reported (Bishop et al, 2000; Muzio et al, 2002; Bishop et al, 2002). By the same token, reduction in Pax6 concentration decreases effectively the output signal $f$ (Fig. 6a), which in turn shifts anteriorly the concentrations of the molecules A, B and C (Fig. 6b). This rearrangement pushes TC axons more into anterior end to new equilibrium positions (Fig. 6c) that leads to areas shift in this direction (Figs. 6d,e).

The action of FGF8 is only slightly different. When we increase the expression level of FGF8 by amplifying its amplitude $A_{f g f}$ and its range, we also increase the signal $f$ that leads to posterior shift in expression levels of the molecules A, B and C (Fig. 7a). 
As a consequence of this, TC connectivity (Fig. 7b) and cortical areas (Fig. 7c) shift posteriorly, similarly to what happens in the case of reduced Emx2. In contrast, if we decrease the expression of FGF8 by decreasing $A_{f g f}$ and the range of its expression, then we obtain pattern shifting in the opposite direction by the same mechanism as above (Figs. 7d,e,f). Both types of shifting have been experimentally observed recently (Fukuchi-Shimogori and Grove, 2001, 2003; Garel et al, 2003).

\section{Generation of two separate S1 fields.}

In experiments with an extra source of FGF8 at the posterior end, in some cases, a second entirely separate S1 barrel field can be generated (Fukuchi-Shimogori and Grove, 2001). We can mimic this effect of "mirror symmetry" in our model (Figs. 8a,b). The key observation is that by modifying the expression level of FGF8, one changes the spatial shape of the signal $f(x)$. Without the second ectopic source of FGF8 at the posterior end, this function decays monotonically from the anterior to the posterior end. However, when this second source is present, the function $f(x)$ can have a minimum at central part under some conditions (Fig. 8c). This happens if the diffusion range of the ectopic FGF8 is not too large and if its amplitude is sufficiently strong to overcome inhibition of Emx2 in the posterior end. From eq. (9), then it follows that $\rho_{B}(x)$ is expressed at high levels at two separate central locations which partly overlap (Fig. 8d). Thus, instead of growing to one central location, axons of type $i=3$ grow to two slightly separated locations that correspond to two partly symmetric S1 areas (Figs. 8a,b). However, we are unable, within our approach, to distinguish between two possibilities: one, that the same group of axons target two S1 fields by branching in two 
separate locations, and second, that axons subdivide into two groups, each targeting only one S1 field. These two possibilities await experimental teasing apart.

Surprisingly, this is not the only modification that the second ectopic source of FGF8 brings. It turns out that the whole pattern of both TC connectivity (Fig. 8a) and cortical fields (Fig. 8b) has a mirror symmetry. Area V1 $\left(c_{5}\right)$ is positioned in the gap between the two S1 $\left(c_{3}\right)$ areas, and area M1 $\left(c_{1}\right)$ has two branches: one in the regular anterior end, and another in the posterior end that under normal conditions would be occupied by V1. Also, area $c_{2}$ has two branches, and area $c_{4}$ overlaps S1 and V1. These results can be understood by invoking again Figs. 8c,d and eqs. (8)-(10). The guiding molecule $\mathrm{C}$ that acts as an attractant for axons terminating in $\mathrm{V} 1$ area is expressed now only slightly in the central part due to the modified shape of the signal $f(x)$ (only for $x$ located in the center, $f(x)$ is relatively close to the threshold $\theta_{4}$ ). Axonal branches of type $i=5$ gathered in the center due to attraction to $\mathrm{C}$, are additionally repulsed by two regions of high concentration of B (Fig. 8d), and that effectively pushes them to the gap between the two S1 fields. Similar arguments hold for the M1 $\left(c_{1}\right)$ fields. The molecule A that attracts axons of type 1 terminating in M1 is expressed now not only in the anterior end but also partially in the posterior end (Fig. 8d). This leads to the type $i=1$ axons terminating in each of these locations (Fig. 8a). Similarly for the area $c_{2}$. Axonal branches of this type are the most repulsed by the molecules $\mathrm{B}$ and $\mathrm{C}$, and only weakly repulsed by A. As a result, they grow in the regions where the molecule A has a moderate expression, that is, also in two symmetric locations.

From the above it is apparent that the ectopic FGF8 can profoundly affect the 
architecture of the neocortex by creating and destroying areas at different locations. It would be interesting to verify these predictions experimentally.

\section{Discussion}

In this paper, a mathematical model of the early development of TC connections and cortical area patterning is presented. Cortical patterning is achieved in the model by allowing TC axonal branches to undergo a combination of biased random growth with their mutual competition for neocortical space. The model captures the essential components, which have been experimentally implicated as the important genetic factors (Bishop et al, 2000; Mallamaci et al, 2000; Fukuchi-Shimogori and Grove, 2001, 2003; Bishop et al, 2002; Muzio et al 2002; Garel et al 2003). Its strength is in its ability to reproduce several recent experiments within a single theoretical framework, and also in its potential to predict the outcomes of some experiments. In this regard, one of the tests of this model can be experimental verification of the theoretical findings related to duplication of S1 field. In particular, to test (i) if cells in the gap between two symmetric S1 fields really acquire the properties typical for neurons in the visual cortex, and (ii) if cells in the posterior end acquire properties typical for motor neurons.

Other tests of the model that are perhaps more feasible experimentally could include the influence of the transcription factors Emx2 and Pax6, and FGF8 on the guidance molecules. If the guiding molecules $\mathrm{A}, \mathrm{B}$, and $\mathrm{C}$ really correspond to ephrins $\mathrm{A} 4, \mathrm{~A} 5$, and B3, respectively, then by manipulating Emx2, Pax6, and FGF8 one should be able to observe changes in ephrins concentrations. Specifically, in shifting experiments, 
we predict that for Emx2 mutants the concentrations of all three ephrins should shift posteriorly (Fig. 5b), and for Pax6 mutants they should shift anteriorly (Fig. 6b) as compared to the wild-type case. Partial confirmation of this prediction is provided by the data of Bishop et al (2002), who found that ephrin-A5 concentration shifts posteriorly in Emx2 mutants and it shifts anteriorly in Pax6 mutants. In the case of FGF8, we predict that when FGF8 is overexpressed in the anterior pole, then all ephrins should shift posteriorly (Fig. 7a), and when FGF8 is underexpressed, they should shift in the opposite direction (Fig. 7d). In the case of two sources of FGF8 present at the two poles, a crucial experiment would be to verify Fig. 8d. In particular, the model predicts that the molecule A (ephrin-A4) should be expressed not only in the anterior end (regular location) but also in the posterior end, and additionally that the molecule C (ephrin-B3) should be minimally expressed in the center of the cortex instead of the posterior end. Such experiment would prove or disprove the correctness of our assumption on the relationship between FGF8 and the axon guidance molecules.

Our model is robust to changes in the interaction parameters between transcription factors $\left(v_{1}, v_{2}, w_{1}, w_{2}\right)$. However, these parameters cannot be too small, since then the effects of area shifting are small too. In general, the larger these parameters the more pronounced the shiftings.

The most crucial interactions in the model are the chemo-interactions between the axon guidance molecules and TC axons, i.e. the matrix $\gamma$. Together with axonal competition (eq. (3)), the elements of this matrix control the location and border sharpness of cortical areas. The results do not depend dramatically on their precise values and se- 
lectivity in sign, but rather they depend on their relative magnitudes. In order to obtain the correct TC projections in wild-type conditions, it is essential to have the following pattern in the chemo-interactions: axons that are supposed to terminate in the anterior part of the cortex should be strongly repulsed by the guidance molecules located in the posterior end, moderately repulsed by the centrally located guidance molecules, and either attracted or very weakly repulsed by the anterior guidance molecules. Analogically, for the axons that are supposed to terminate in the posterior part, the reverse pattern of interactions is necessary, i.e. these axons should be strongly repulsed by the anterior guidance molecules, moderately repulsed by the central guidance molecules, and either attracted or very weakly repulsed by the posterior molecules. For the axons that are supposed to terminate in the central part of the cortex, it is important that they interact with the posterior and anterior guidance molecules in a symmetric manner, i.e. they should be repulsed by them with approximately the same strength. The larger this strength the more confined the axonal projections are to the central part. The interaction with the central guidance molecules is not very important, i.e. it can be attractive (of any strength) or repulsive (but the strength cannot exceed the repulsion from the lateral molecules).

In this paper we assumed that the axon guidance molecules act selectively such that they repulse some classes of TC axons and attract other. However, this assumption is not crucial for the results. Even, if it turns out that the net effect of chemo-interaction between cortical ephrins (and possibly other guidance molecules) and TC axons is repulsion, the appropriately modified interaction matrix would not qualitatively change 
the results presented here. As an example, in Fig. 9 we show a spatial profile of TC connectivity when the matrix $\gamma$ has only negative elements but with the pattern of their inter-relationships discussed above. In this case one can still generate separate areas, although with much less sharp borders. They shift as Emx2, Pax6 or FGF8 are up- or downregulated, similarly as it happens in the case with the interaction matrix that has negative and positive elements (Fig. 9b). Also, one can generate a mirror symmetry effect when two sources of FGF8 are present at two separate poles (Fig. 9c).

It is interesting to note that an analog of the mirror symmetry effect occurs also in the retino-tectal system (Fig. 2c,d in McLaughlin et al, 2003). This takes place in mice with ephrin-A5 and ephrin-A2/ephrin-A5 mutants. There is some analogy with our case of the double FGF8 source, because in our model its second ectopic source also modifies the guiding molecules, especially their posterior distributions.

In the model we consider, motivated by experimental data, the three types of the guidance molecules. However, one can obtain qualitatively similar results with only one type of guiding molecule located primarily either in the posterior or anterior part of the cortex. Regionalization of the cortex then would be obtained by appropriately choosing the elements of the matrix $\gamma$ in the similar way as we did in this paper. The seemingly uneconomical number of the axon guidance molecules chosen by nature may be necessary for a finer targeting of TC axons within cortical areas.

Our model is the first study, which takes into account genetic factors (Emx2, Pax6, FGF8) and their influence on the gradients of the guiding molecules. However, some aspects of the axon guidance part have been modeled previously in the context of retino- 
tectal projections (Prestige and Willshaw, 1975; Fraser and Hunt, 1980; Whitelaw and Cowan, 1981; Gierer, 1983). The model of Whitelaw and Cowan (1981) puts a greater emphasis on synaptic/neural activity than on guidance molecules. The model of Gierer (1983) seems to be somewhat abstract; it uses minimization procedure of some abstract function related to retinal and tectal gradients in order to obtain topographic mapping. The axonal guidance part of our model is closest in spirit to the models of Prestige and Willshaw (1975), and Fraser and Hunt (1980), which also used the idea of axonal competition. However, there are several significant differences between these models and our model. Our main objective was to generate topographic arealization with sharp borders, while their goal was to generate continuous topography without areas. In terms of mathematics, our model uses the reaction kinetics equations (1-4) on a population level, while Prestige and Willshaw (1975) use algorithmic approach with a discrete time on a single axon/neuron level. Fraser and Hunt (1980), on the other hand, impose several constraints on axonal dynamics that guide them to the correct locations. Also, we consider explicitly the forces between TC axons and the guidance molecules, and additionally stochastic branching effects employed by the diffusion term (see, Eq. (4)); these features are absent in their models.

The present model can be also modified by including more biophysical details, more signaling molecules in the pathway, more pathways, and cross-interactions between pathways, if necessary. For example, it is likely that more signaling molecules are involved in regulating axon guidance molecules and that the simple scheme in Fig. 1 will be expanded as more experimental data becomes available. Other candidates playing 
a role similar to FGF8 but providing positional information from another cortical end, could be WNT and BMP molecules located on the cortical hem (O'Leary and Nakagawa, 2002; Grove and Fukuchi-Shimogori, 2003). However, if those molecules act in coordination with FGF8, then it is unlikely that such an expanded model would qualitatively change the present results.

Throughout the paper, we assume that genetic effects determine the very early stage of cortical development and set the basic parcelation of the neocortex (Miyashita-Lin et al, 1999; Nakagawa et al, 1999). Thus, we model only the early cortical development, before and right after the arrival of TC input. Later stages of the development are probably controlled by TC input (i.e. molecules diffused by arrived TC axons), and by activities of neurons and their synapses (Katz and Shatz, 1996), and therefore require a different approach. However, it is likely that those late stage activities only refine that basic patterning plan set by genetic factors (O'Leary and Nakagawa, 2002; LopezBendito and Molnar, 2003). In this respect, it is probable that neural activities can additionally reduce a partial overlap of the areas that is due to molecules diffusion (e.g. see Fig. 4c). Also those axons/branches diffusing around the cortex that do not establish connections, will likely die out at later stages of the development. That effect is not included in our model.

\section{Acknowledgments}

We thank Elizabeth Grove for comments on a draft of this paper. The work was supported by the Sloan-Swartz fellowship at Caltech (J.K.) and the National Science Foundation (G.B.E.). 


\section{References}

Bishop, K.M., Goudreau, G., and O'Leary, D.D. (2000). Regulation of area identity in the mammalian neocortex by Emx2 and Pax6. Science 288, 344-349.

Bishop, K.M., Rubenstein, J.L.R., and O'Leary, D.D. (2002). Distinct actions of Emx1, Emx2 and Pax6 in regulating the specification of areas in the developing neocortex. J. Neurosci. 22, 7627-7638.

Cohen-Tannoudji, M., Babinet, C., and Wassef, M. (1994). Early determination of a mouse somatosensory cortex marker. Nature 368, 460-463.

Feldheim, D.A., et al. (2000). Genetic analysis of ephrin-A2 and ephrin-A5 shows their requirement in multiple aspects of retinocollicular mapping. Neuron, 25, 563-574.

Finlay, B.L., and Darlington, R.B. (1995). Linked regularities in the development and evolution of mammalian brains. Science 268, 1578-1584.

Fraser, S.E., and Hunt, R.K. (1980). Retinotectal specificity: models and experiments in search of a mapping function. Annu. Rev. Neurosci. 3, 319-352.

Fukuchi-Shimogori, T., and Grove, E.A. (2001). Neocortex patterning by the secreted signaling molecule FGF8. Science 294, 1071-1074.

Fukuchi-Shimogori, T., and Grove, E.A. (2003). Emx2 patterns the neocortex by regulating FGF positional signaling. Nature Neurosci. 6, 825-831.

Garel, S., Huffman, K.J., and Rubenstein, J.L.R. (2003). Molecular regionalization of the neocortex is disrupted in FGF8 hypomorphic mutants. Development 130, 19031914.

Gierer, A. (1983). Model for the retino-tectal projection. Proc. R. Soc. Lond. B 218, 
$77-93$.

Goodhill, G.J., and Richards, L.J. (1999). Retinotectal maps: molecules, models and misplaced data. Trends Neurosci. 22, 529-534.

Grove, E.A., and Fukuchi-Shimogori, T. (2003). Generating the cerebral cortical area map. Annu. Rev. Neurosci. 26, 355-380.

Hofman, M.A. (1989). On the evolution and geometry of the brain in mammals. Prog. Neurobiol. 32, 137-158.

Karbowski, J. (2003). How does connectivity between cortical areas depend on brain size? Implications for efficient computation. J. Comput. Neurosci. 15, 347-356.

Katz, L.C., and Shatz, C.J. (1996). Synaptic activity and the construction of cortical circuits. Science 274, 1133-1138.

Knoll, B., and Drescher, U. (2002). Ephrin-As as receptors in topographic projections. Trends Neurosci. 25, 145-149.

Krubitzer, L. (1995). The organization of neocortex in mammals: Are species differences really so different? Trends Neurosci. 18, 408-417.

Krubitzer, L., and Huffman, K.J. (2000). Arealization of the neocortex in mammals: genetic and epigenetic contributions to the phenotype. Brain Behav. Evol. 55, 322-335. Lopez-Bendito, G., and Molnar, Z. (2003). Thalamo-cortical development: How are we going to get there? Nature Rev. Neurosci. 4, 276-289.

Mackarehtschian, K., Lau, C.K., Caras, I., and McConnel, S.K. (1999). Regional differences in the developing cerebral cortex revealed by Ephrin - A5 expression. Cereb. Cortex 9, 601-610. 
Mallamaci, A., Muzio, L., Chan, C.H., Parnavelas, J., and Boncinelli, E. (2000). Area identity shifts in the early cerebral cortex of $E m x 2^{-/-}$mutant mice. Nature Neurosci. 3, 679-686.

Mann, F., Peuckert, C., Dehner, F., Zhou, R., and Bolz, J. (2002). Ephrins regulate the formation of terminal axonal arbors during the development of thalamocortical projections. Development 129, 3945-3955.

McLaughlin, T., Hindges, R., and O'Leary, D.D.M. (2003). Regulation of axonal patterning of the retina and its topographic mapping in the brain. Curr. Opin. Neurobiol. $13,57-69$.

Miyashita-Lin, E.M., Hevner, R., Wassarman, K.M., Martinez, S., and Rubenstein, J.L.R. (1999). Early neocortical regionalization in the absence of thalamic innervation. Science 285, 906-909.

Murray, J.D. (1993). Mathematical Biology. Springer-Verlag, Berlin, pp. 109-139.

Muzio, L. et al (2002). Emx2 and Pax6 control regionalization of the pre-neuronogenic cortical primordium. Cereb. Cortex 12, 129-139.

Nakagawa, Y., Johnson, J.E., and O’Leary, D.D.M. (1999). Graded and areal expression patterns of regulatory genes and cadherins in embryonic neocortex independent of thalamocortical input. J. Neurosci. 19, 10877-10885.

Nauta, W.J.H., and Feirtag, M. (1986). Fundamental Neuroanatomy. (Freeman, New York).

Northcutt, R.G., and Kaas, J.H. (1995). The emergence and evolution of mammalian neocortex. Trends Neurosci. 18, 373-379. 
O’Leary, D.D.M. (1989). Do cortical areas emerge from a protocortex? Trends Neurosci. $12,400-406$.

O'Leary, D.D.M., Yates, P.A., McLaughlin, T. (1999). Molecular development of sensory maps: representing sights and smells in the brain. Cell 96, 255-269.

O’Leary, D.D.M., and Nakagawa, Y. (2002). Patterning centers, regulatory genes and extrinsic mechanisms controlling arealization of the neocortex. Curr. Opin. Neurobiol. $12,14-25$.

Prestige, M.C., and Willshaw, D.J. (1975). On a role for competition in the formation of patterned neural connexions. Proc. Roy. Soc. Lond. B 190, 77-98.

Rakic, P. (1988). Specification of cerebral cortical areas. Science 241, 170-176.

Simon, D.K., and O'Leary, D.D.M. (1992). Development of topographic order in the mammalian retinocollicular projection. J. Neurosci. 12, 1212-1232.

Sperry, R.W. (1963). Chemoaffinity in the orderly growth of nerve fibers patterns and connections. Proc. Natl. Acad. Sci. USA 50, 703-710.

Takemoto, M., et al (2002). Ephrin-B3-EphA4 interactions regulate the growth of specific thalamocortical axon populations in vitro. Eur. J. Neurosci. 16, 1168-1172.

Uziel, D. et al (2002). Miswiring of limbic thalamocortical projections in the absence of ephrin-A5. J. Neurosci. 22, 9352-9357.

Vanderhaeghen, P. et al (2000). A mapping label required for normal scale of body representation in the cortex. Nature Neurosci. 3, 358-365.

Whitelaw, V.A., and Cowan, J.D. (1981). Specificity and plasticity of retinotectal connections: a computational model. J. Neurosci. 1, 1369-1387. 
Wolpert, L. (1969). Positional information and spatial pattern of cellular differentiation. J. Theor. Biol. 25, 1-47.

Wolpert, L. (1996). One hundred years of positional information. Trends Genet. 12, 359-364.

Yates, P.A., Roskies, A.L., McLaughlin, T., and O'Leary, D.D.M. (2001). Topographicspecific axon branching controlled by ephrin-As is the critical event in retinotectal map development. J. Neurosci. 21, 8548-8563.

Yates, P.A., Holub, A.D., McLaughlin, T., Sejnowski, T.J., and O’Leary, D.D.M. (2004). Computational modeling of retinotopic map development to define contributions of EphA-EphrinA gradients, axon-axon interactions, and patterned activity. J. Neurobiol. $59,95-113$. 


\section{Figure Captions}

Fig. 1

Schematic diagram depicting the pathway influencing the axon guidance molecules A, B, and C. The transcription factors Emx2 and Pax6 repress each other with strengths $v_{1}$ and $v_{2}$. Emx2 represses FGF8 with strength $w_{1}$, and FGF8 represses Emx2 with strength $w_{2}$. The renormalized expression concentration $f$ of FGF8 or some other signal activated by it serves as a morphogen for the guiding molecules A, B, and C.

Fig. 2

Stationary spatial profiles of the signal $f$ and the guiding molecules under normal conditions (wild type). (A) Renormalized concentration $f(x)$ of FGF8. (B) Concentrations of the guiding molecules A, B, and C. Dashed line represents the concentration of A, solid line corresponds to B, and dashed-dotted line represents the molecule C. Parameters used: $A_{e m x}=1.34, A_{p a x}=1.4, A_{f g f}=0.9, \zeta_{e m x}=25.6, \zeta_{\text {pax }}=27.3, \zeta_{f g f}=26.4$, $w_{1}=2.4, w_{2}=2.1, v_{1}=2.6, v_{2}=2.7, \gamma_{A 1}=1.6, \gamma_{A 2}=-0.4, \gamma_{A 3}=-2.21, \gamma_{A 4}=-2.1$, $\gamma_{A 5}=-2.45, \gamma_{B 1}=-0.6, \gamma_{B 2}=-0.5, \gamma_{B 3}=0.4, \gamma_{B 4}=-0.5, \gamma_{B 5}=-1.0, \gamma_{C 1}=-2.9$, $\gamma_{C 2}=-2.5, \gamma_{C 3}=-2.23, \gamma_{C 4}=-0.6, \gamma_{C 5}=1.7, \theta_{1}=0.77, \theta_{2}=0.5, \theta_{3}=0.39$, $\theta_{4}=0.08, \kappa_{A}=0.58, \kappa_{B}=0.9, \kappa_{C}=0.55, \sigma_{A}=\sigma_{B}=\sigma_{C}=0.2$. Parameters for the dynamics of TC connections: $D=0.1, L=40$, grid size $d x=0.25, \alpha_{i}=3.0, \beta_{i}=3.0$ for $i=1,2,3$.

\section{Fig. 3}

Temporal evolution of the pattern of axonal densities around the cortical surface under normal conditions. (A) Initial distribution, (B) distribution after $t=6$, (C) steady- 
state. Note the emergence of the heterogeneous pattern. Solid line represents the profile of $a_{1}$, dashed-dotted line correspond to $a_{2}$, solid line with open circles represents $a_{3}$, dashed line corresponds to $a_{4}$, and dotted line corresponds to $a_{5}$. Parameters are the same as in Fig. 2.

Fig. 4

Temporal evolution of the pattern of TC connectivity under normal conditions. (A) Initial distribution with no TC connectivity (all lines collapse onto one), (B) distribution after $t=6,(\mathrm{C})$ steady-state. Note the emergence of sharp bordered areas with different axon types. Regions of high values of $c_{1}, c_{3}$, and $c_{5}$ correspond to areas M1, S1, and V1, respectively. Solid line represents the profile of $c_{1}$, dashed-dotted line correspond to $c_{2}$, solid line with open circles represents $c_{3}$, dashed line corresponds to $c_{4}$, and dotted line corresponds to $c_{5}$. (D) Schematic stationary pattern of emerged areas on the cortical surface, corresponding to the TC connectivity in (C). Parameters are the same as in Fig. 2.

\section{Fig. 5}

Stationary spatial profiles of the signal $f$, the axon guidance molecules, axonal densities, TC connectivity, and cortical area pattern when the transcription factor Emx2 is not expressed. For Emx2 mutants the distributions of signal $f(x)(\mathrm{A})$, guiding molecules (B), density of axons (C), TC connectivity (D), and cortical areas (E) all shift posteriorly. In (A) the dashed line corresponds to the control distribution of $f(x)$ from fig. 2A. Parameters are the same as in Fig. 2, except for $A_{e m x}=0$. 
Fig. 6

Stationary spatial profiles of the signal $f$, the axon guidance molecules, axonal densities, TC connectivity, and cortical area pattern when the transcription factor Pax6 is not expressed. For Pax6 mutants the distributions of signal $f(x)(\mathrm{A})$, guiding molecules (B), density of axons (C), TC connectivity (D), and cortical areas (E) all shift anteriorly. In $(\mathrm{A})$ the dashed line corresponds to the control distribution of $f(x)$ from fig. $2 \mathrm{~A}$. Parameters are the same as in Fig. 2, except for $A_{\text {pax }}=0$.

\section{Fig. 7}

The influence of FGF8 on the cortical architecture and guiding molecules. (A), (B), and (C) Overexpression of FGF8. (D), (E), and (F) underexpression of FGF8. Note the opposite shifts of the areas in these cases (compare (B), (C) with (E), (F), respectively). Line convention is the same as in Figs. 2 and 4. Parameters used: (A), (B) $A_{f g f}=1.6$, $\zeta_{f g f}=32.8,(\mathrm{C}),(\mathrm{D}) A_{f g f}=0.6, \zeta_{f g f}=19.0$. Other parameters are the same as in Fig. 2.

Fig. 8

Generation of two partly symmetric S1 areas when two sources of FGF8 are present. (A) Stationary spatial pattern of TC connectivity and (B) corresponding area pattern on the cortical surface. Note the mirror symmetry effect, i.e. not only two S1 areas $\left(c_{3}\right)$ are generated, but also two M1 fields $\left(c_{1}\right)$ are present (one regular, second in the posterior end), and V1 area $\left(c_{5}\right)$ is located in between two S1 areas. (C) Spatial profile of the signal $f$. Note a change of shape and the appearance of a minimum. (D) Stationary concentrations of the guiding molecules. Note that the molecule B is expressed broadly 
and it has two maxima, the molecule A is also partly expressed in the posterior end, and $\mathrm{C}$ is weakly expressed in the central part corresponding to minimum in $\rho_{B}$. The figures are generated by assuming that there is a second source of FGF 8 at the posterior end with the amplitude $A_{f g f}^{\prime}=1.5$ and the range $\zeta_{f g f}^{\prime}=12.0$. Other parameters are the same as in Fig. 2.

\section{Fig. 9}

Stationary spatial patterns of TC connectivity in the case of purely repulsive interactions between guiding molecules and axons. (A) Pattern of TC connectivity under normal conditions. (B) Pattern of TC connectivity with Emx2 mutation, i.e. $A_{e m x}=0$. (C) Pattern of TC connectivity when two sources of FGF8 are present similarly as in Fig. 8. Note a mirror symmetry effect. Parameters used are the same as in Fig. 2 except: $\gamma_{A 1}=-0.08, \gamma_{B 3}=-0.1, \gamma_{C 5}=-0.06$. 


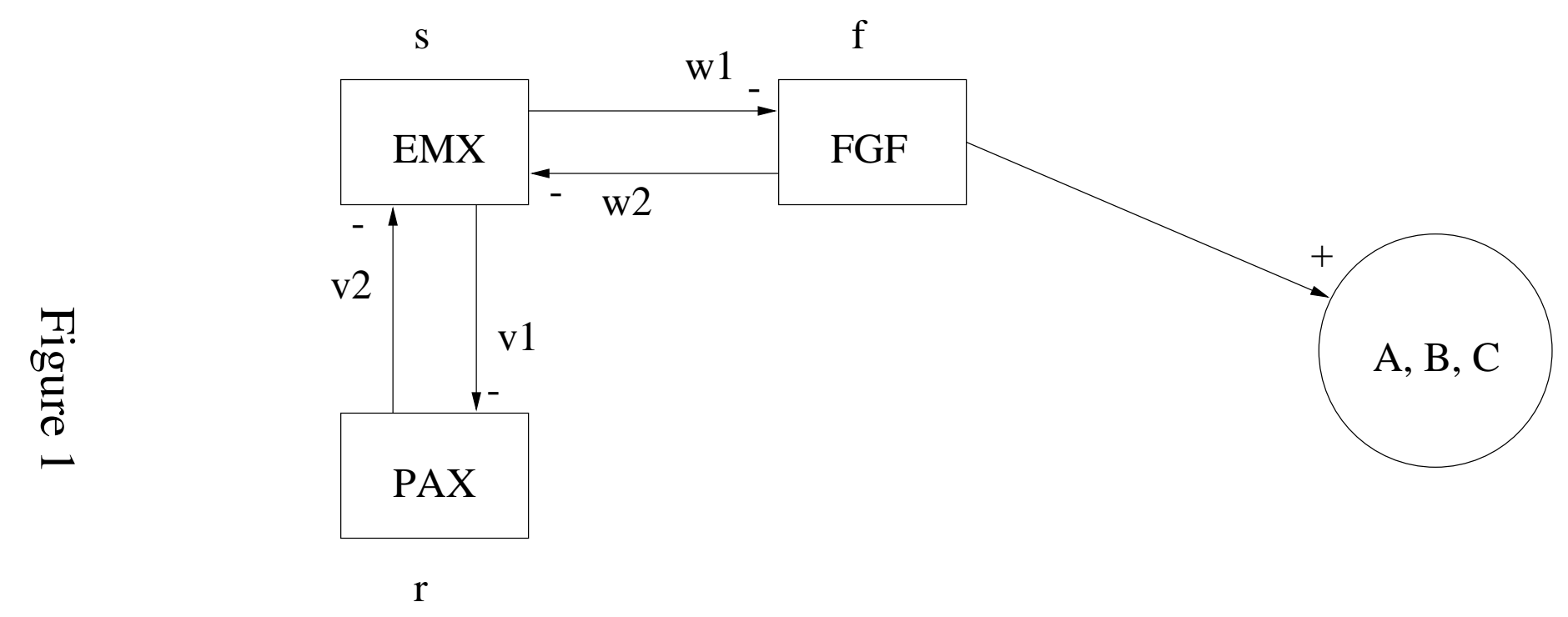




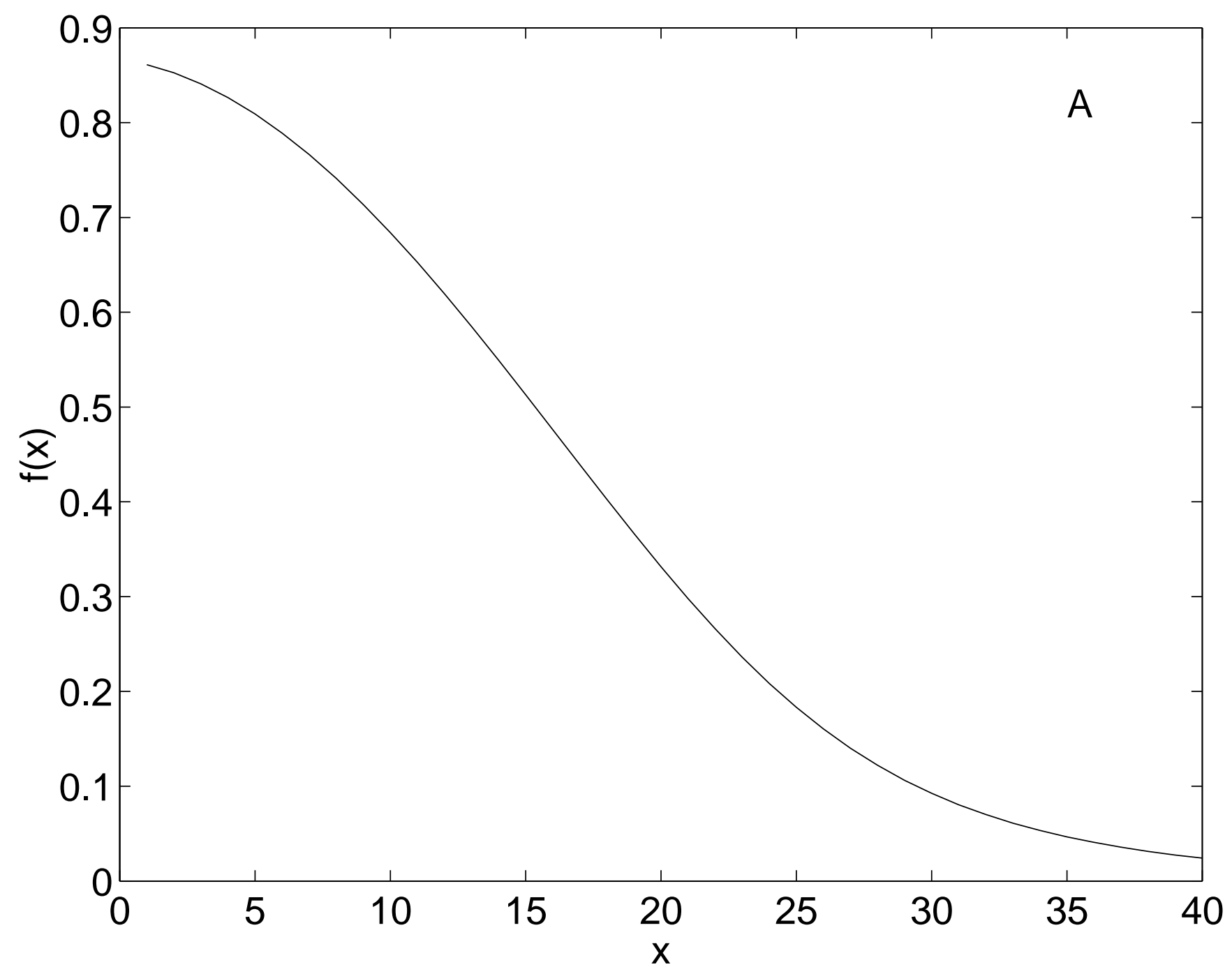

Figure 2A 


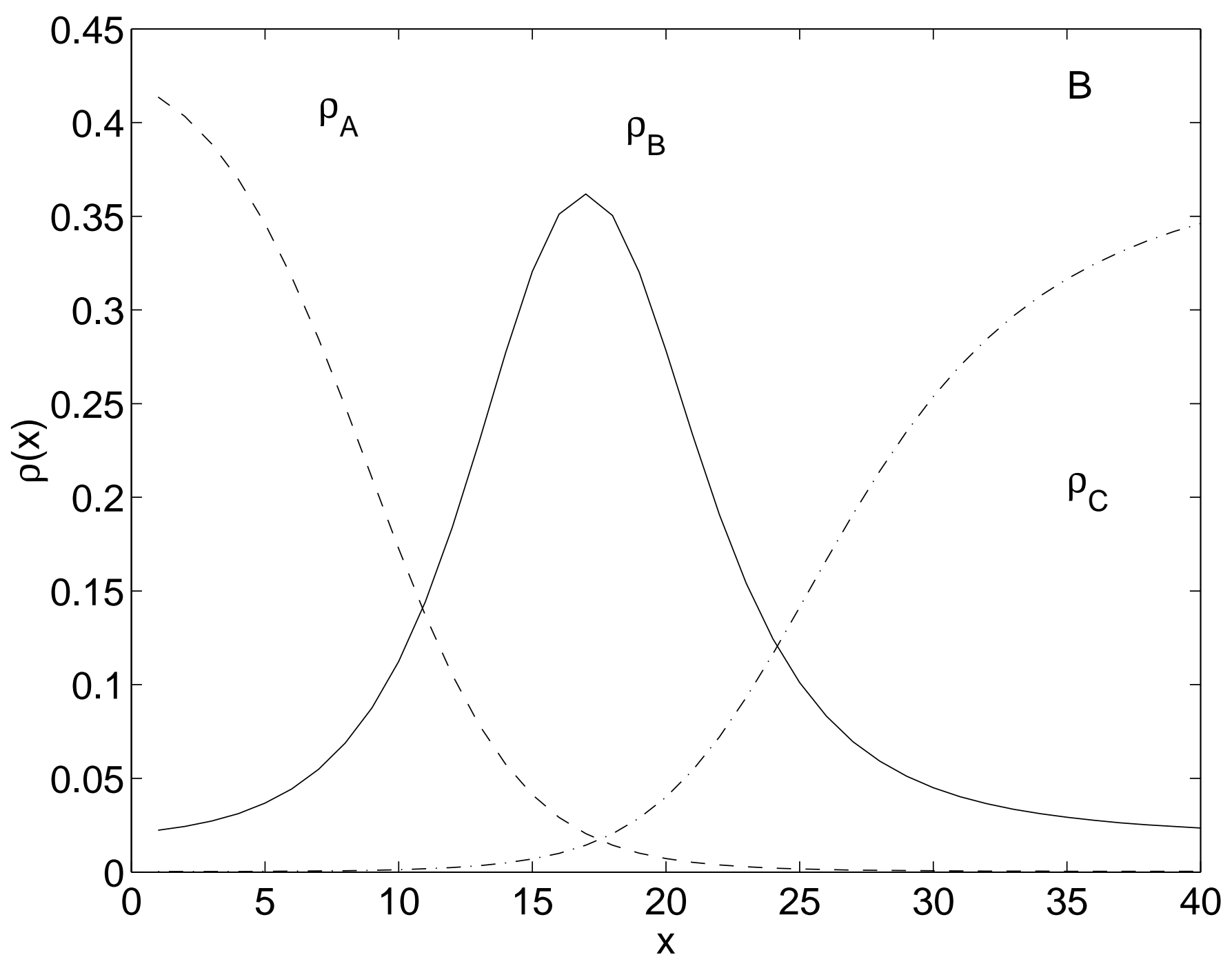

Figure 2B 


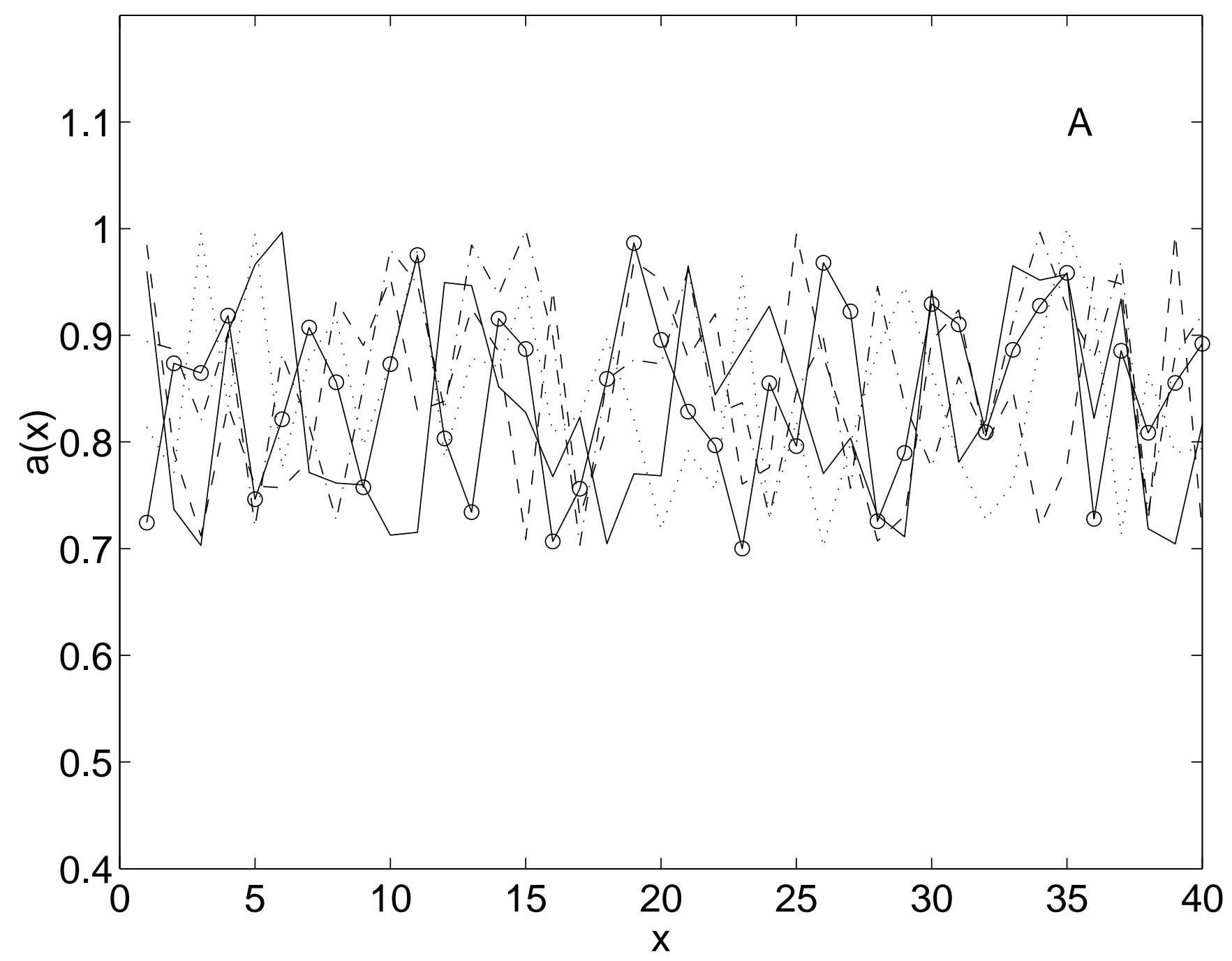

Figure 3A 


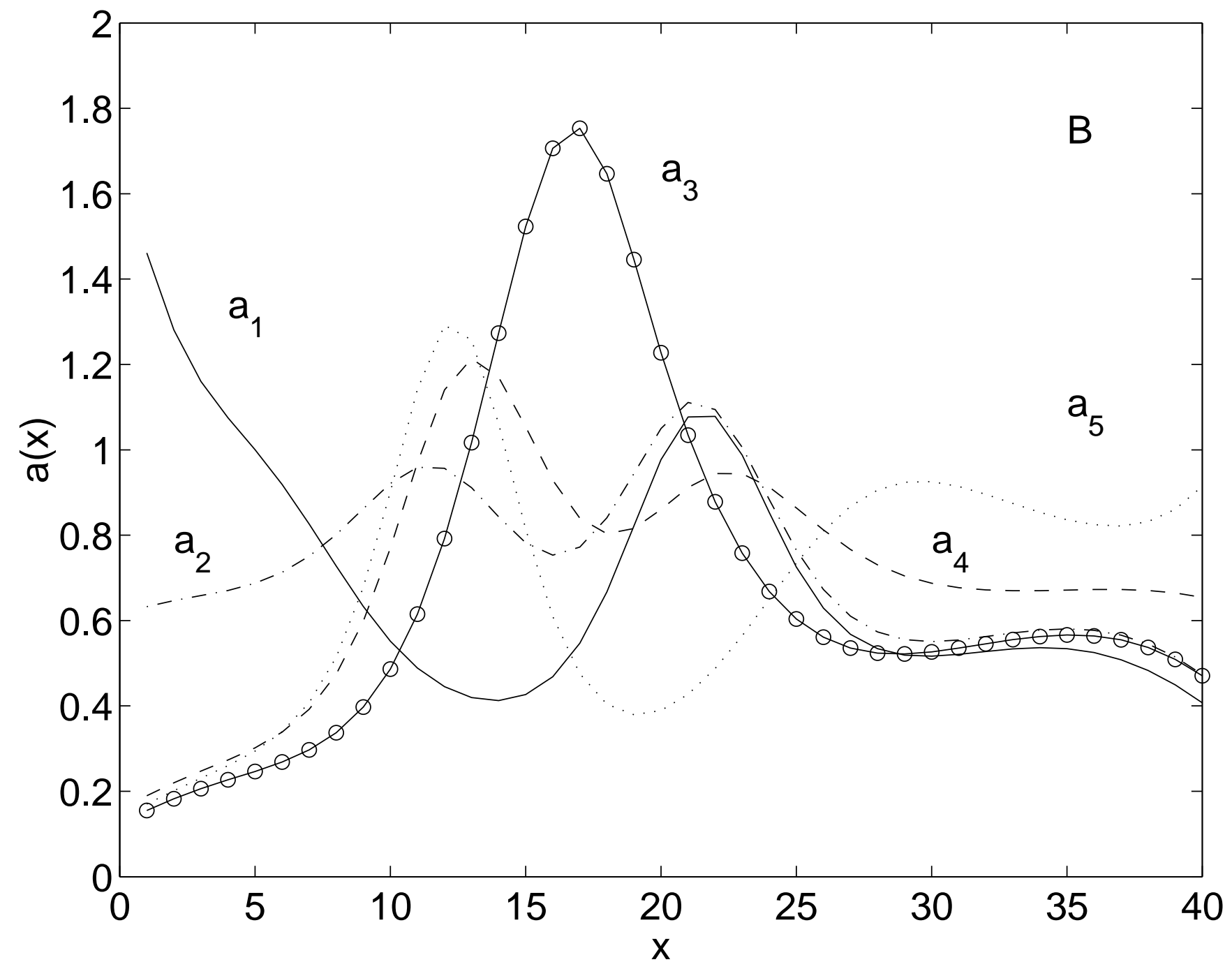

Figure 3B 


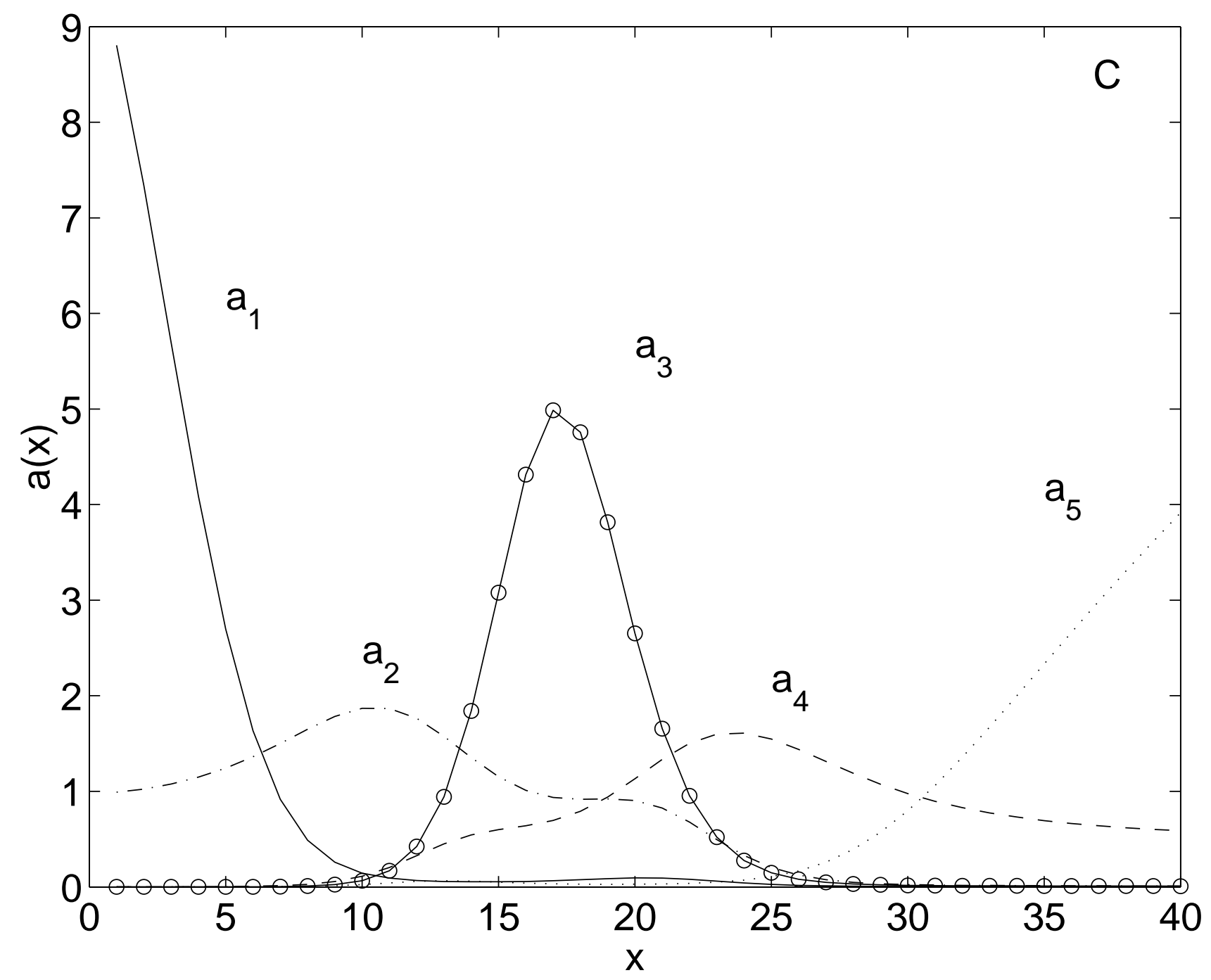

Figure 3C 


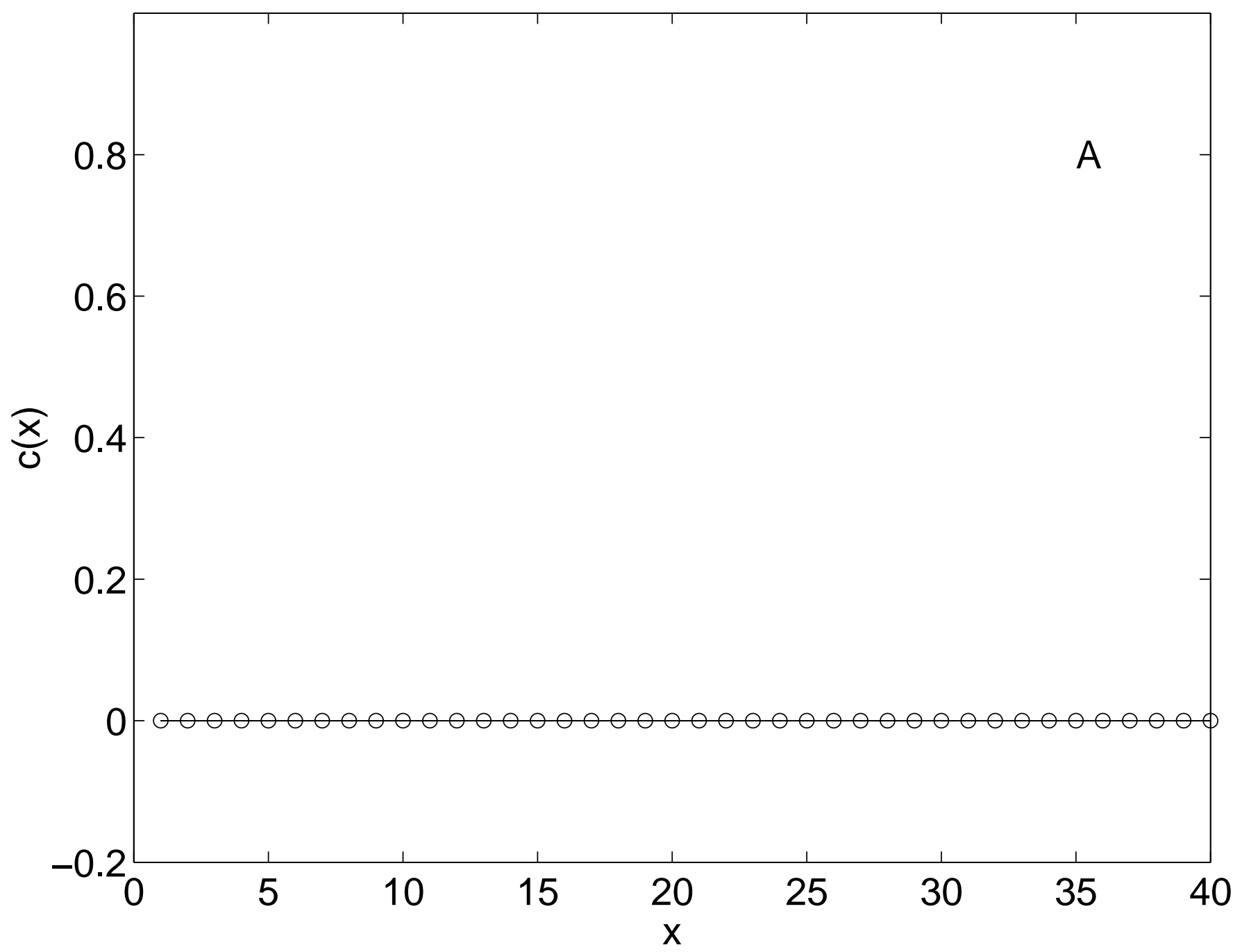

Figure 4A 


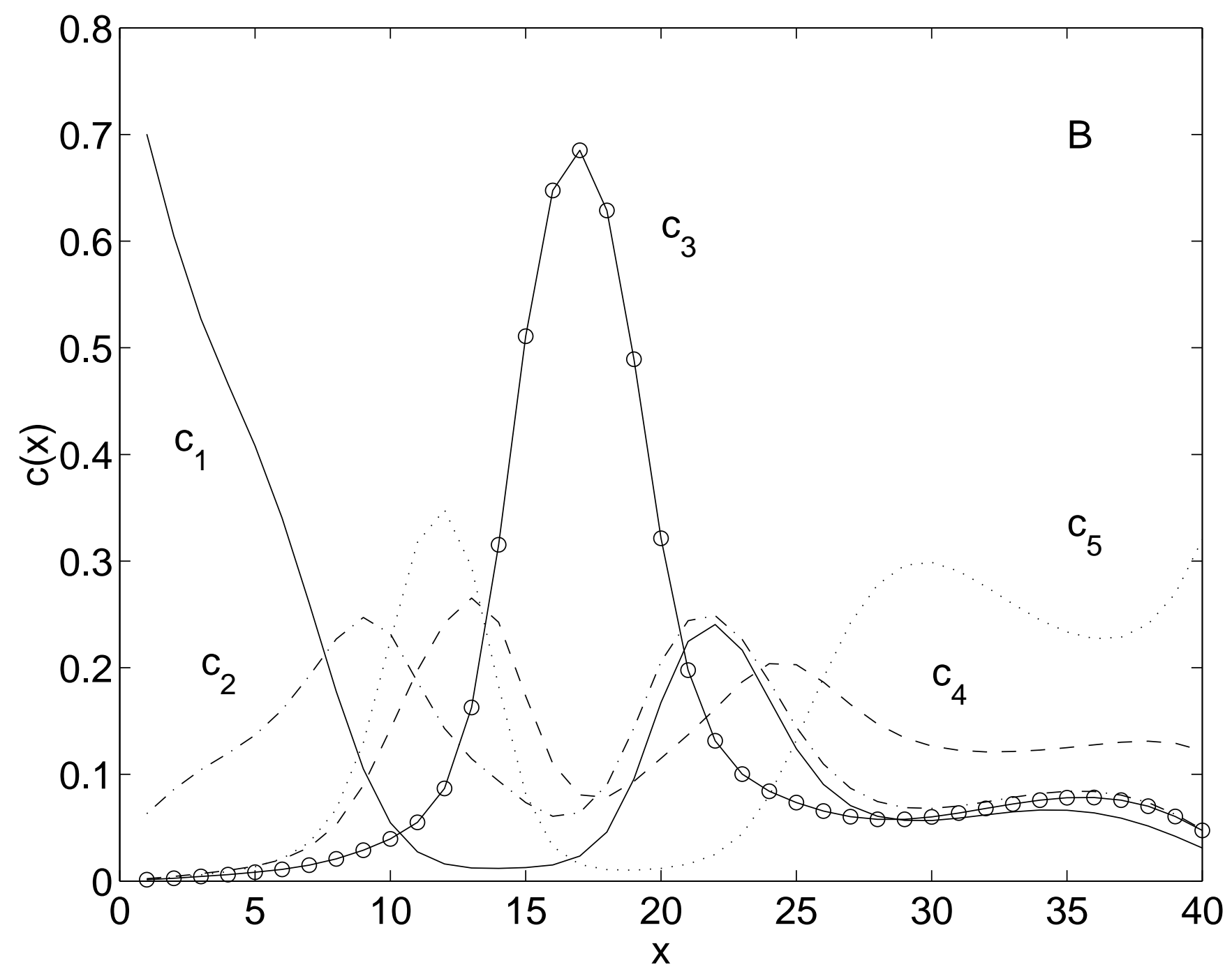

Figure 4B 


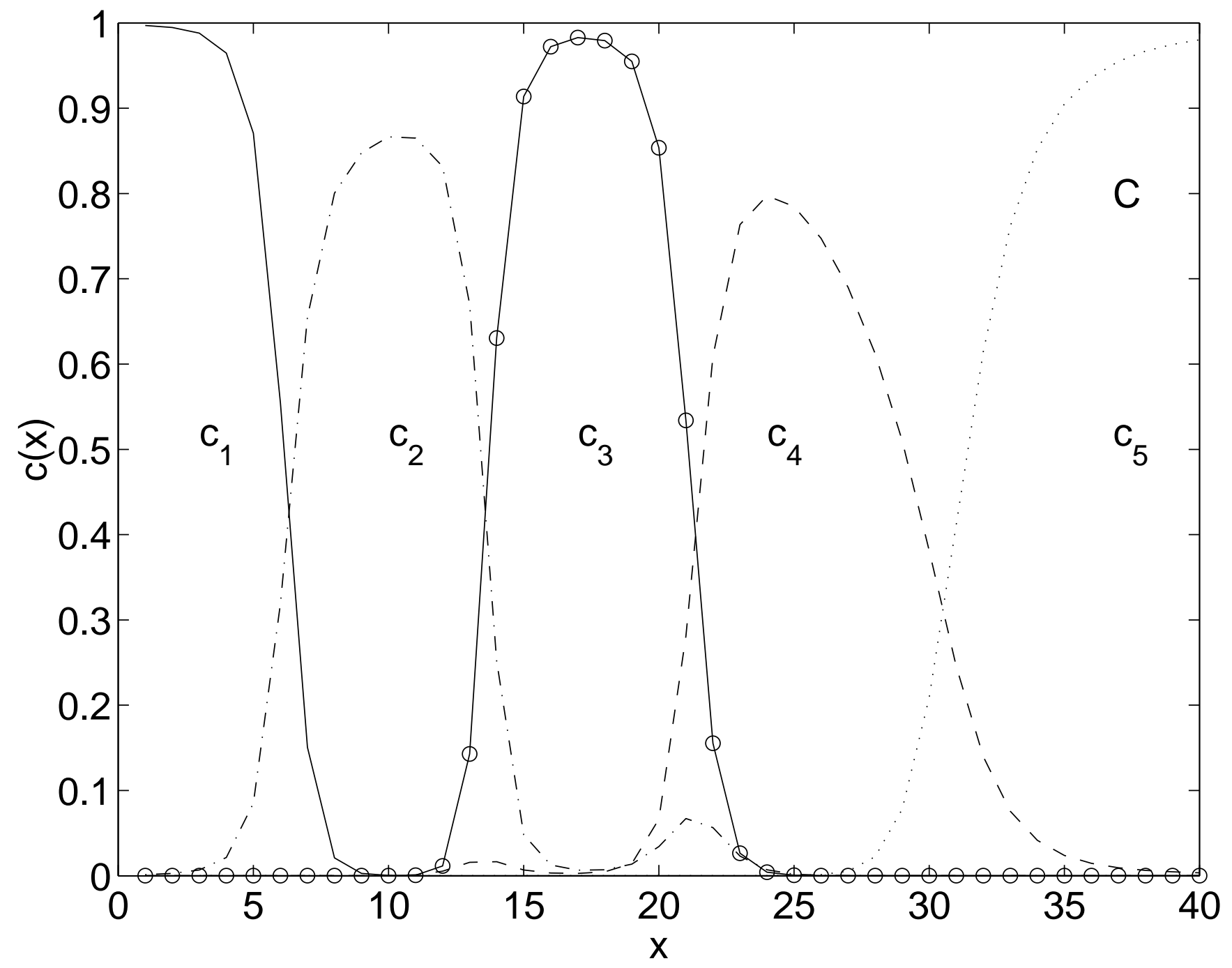

Figure 4C 


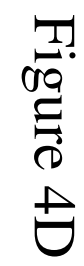

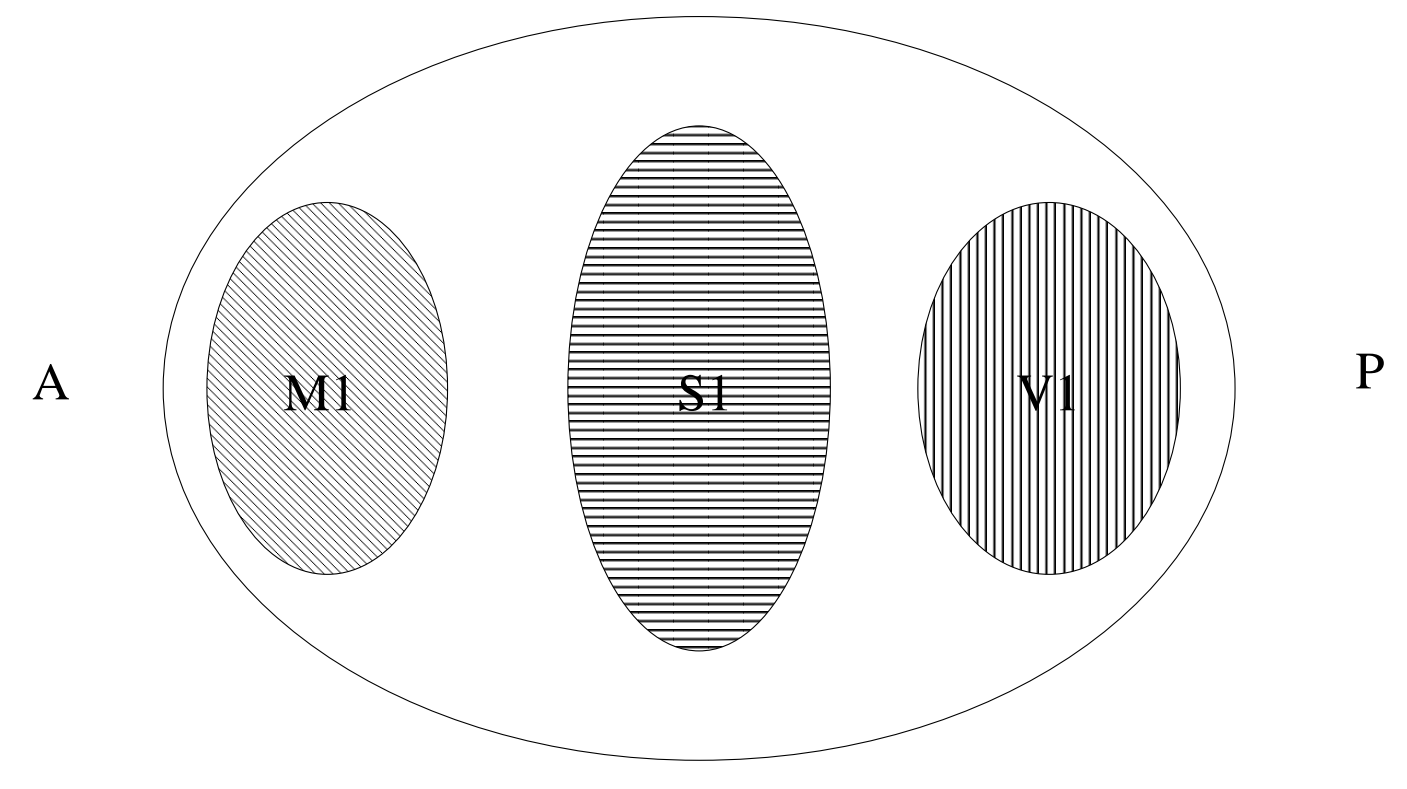




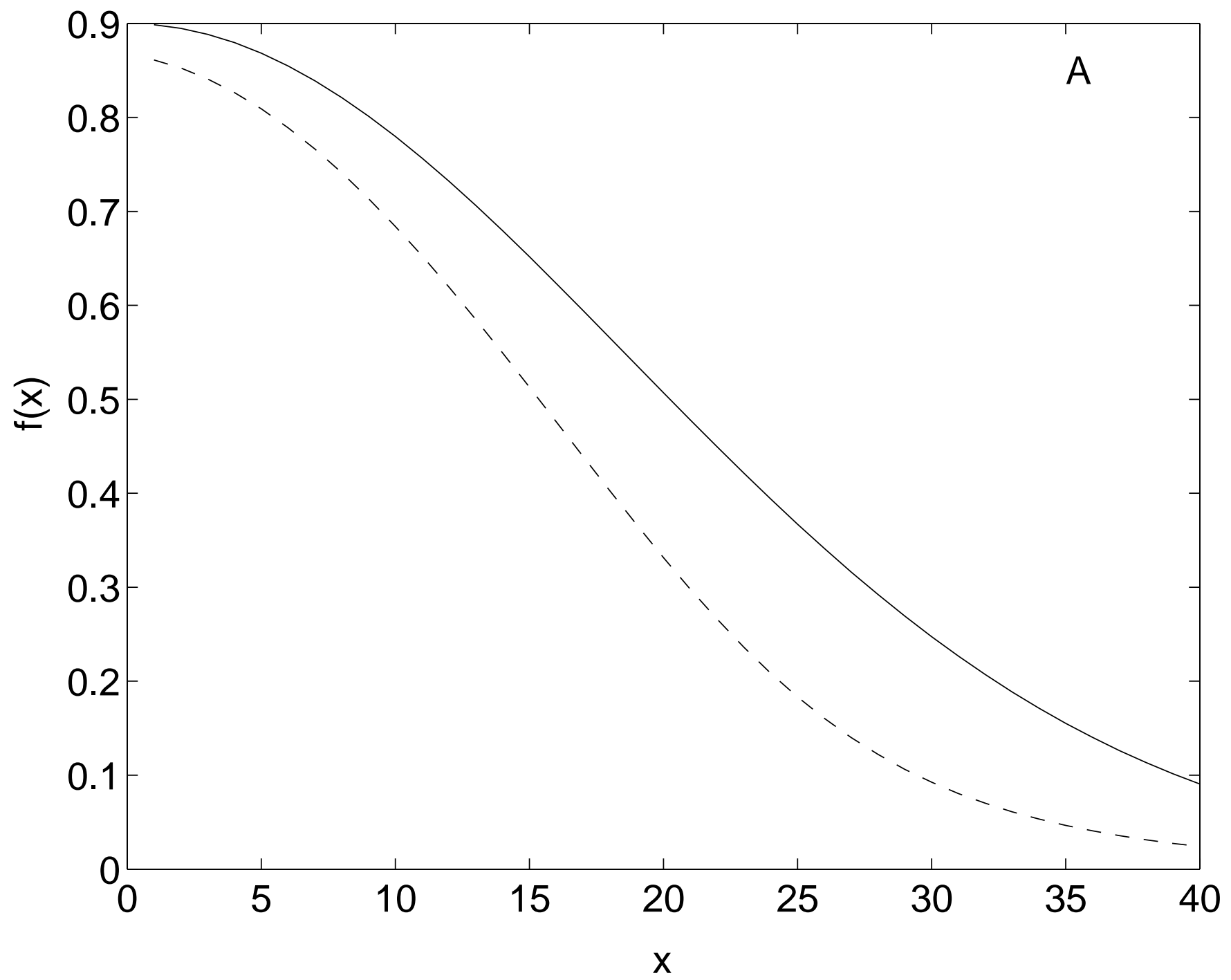

Figure 5A 


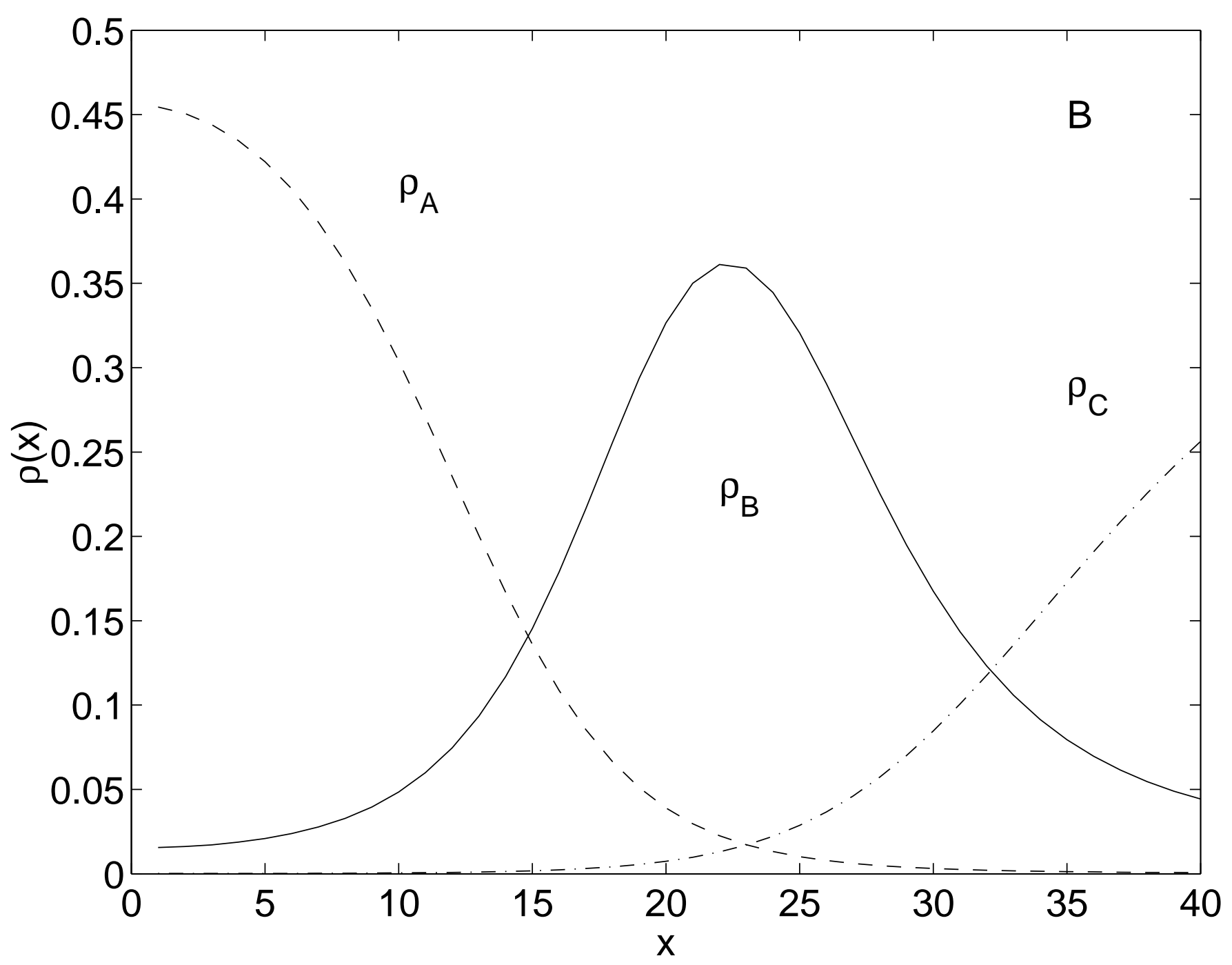

Figure 5B 


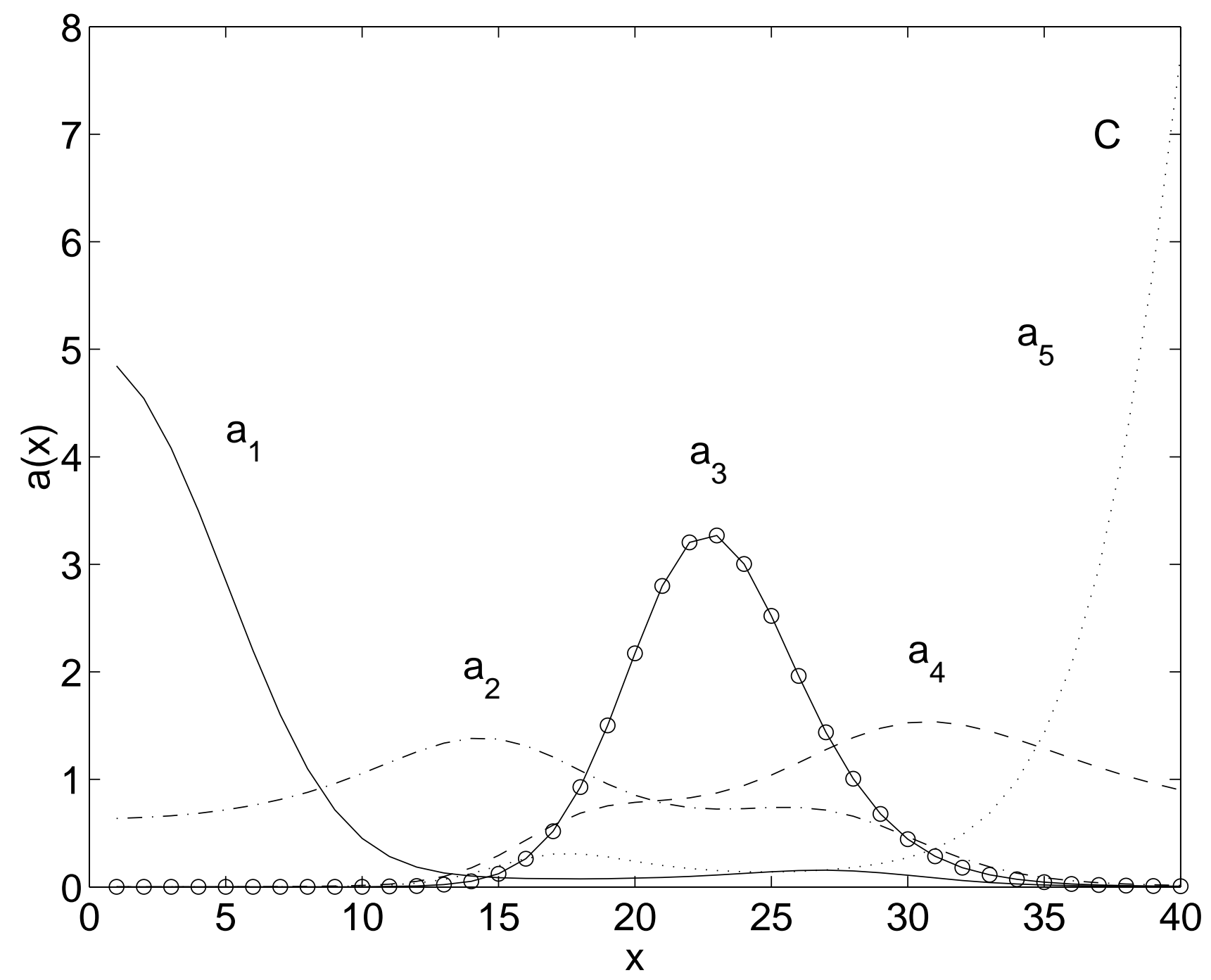

Figure 5C 


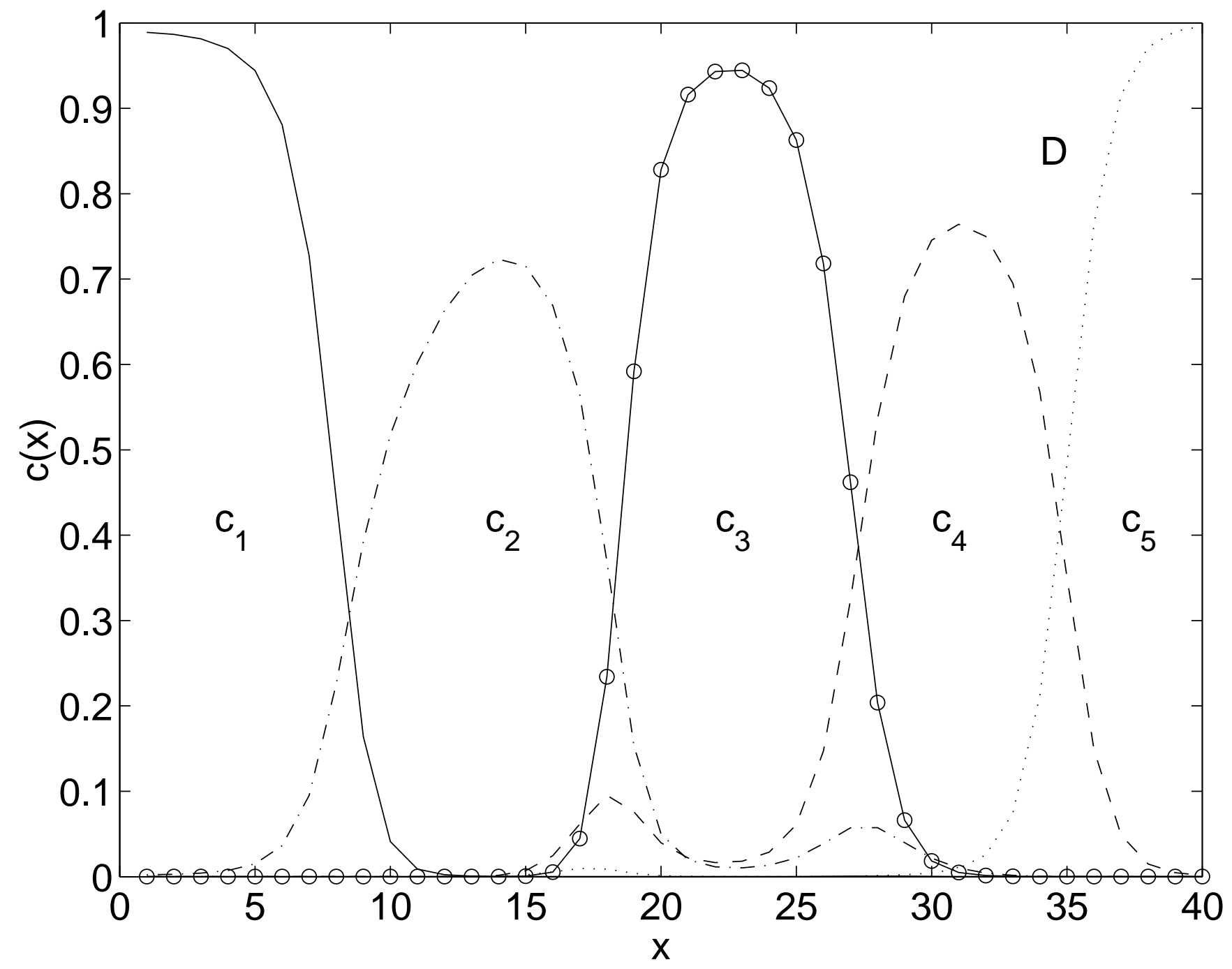

Figure 5D 

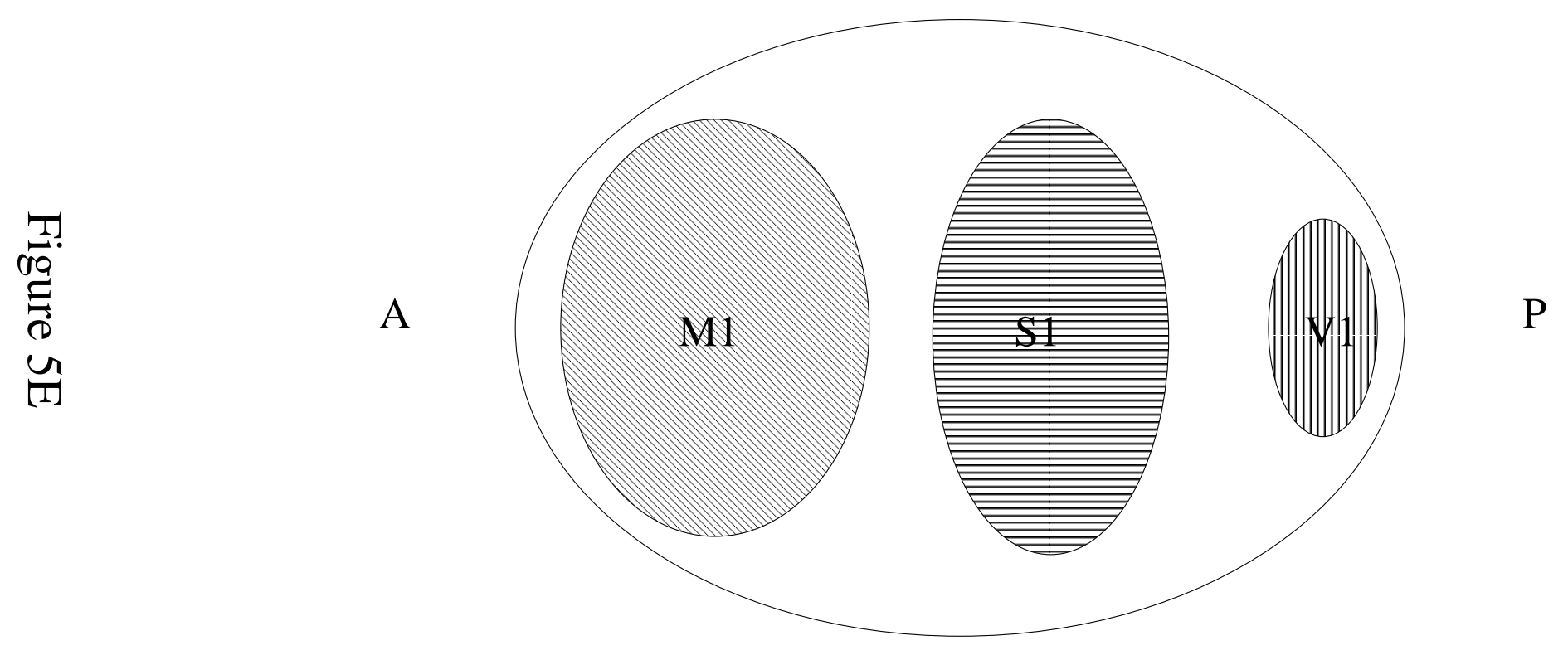


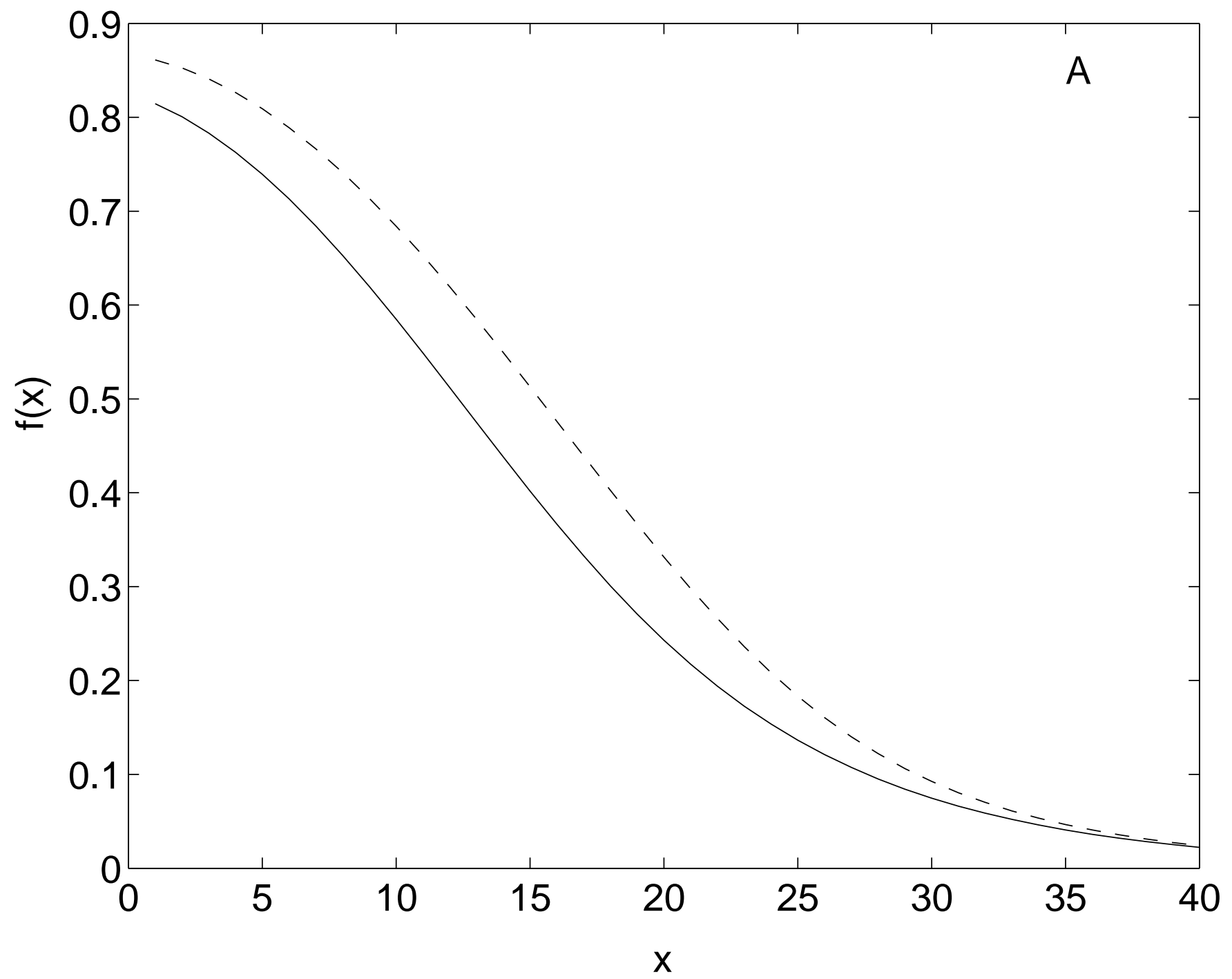

Figure 6A 


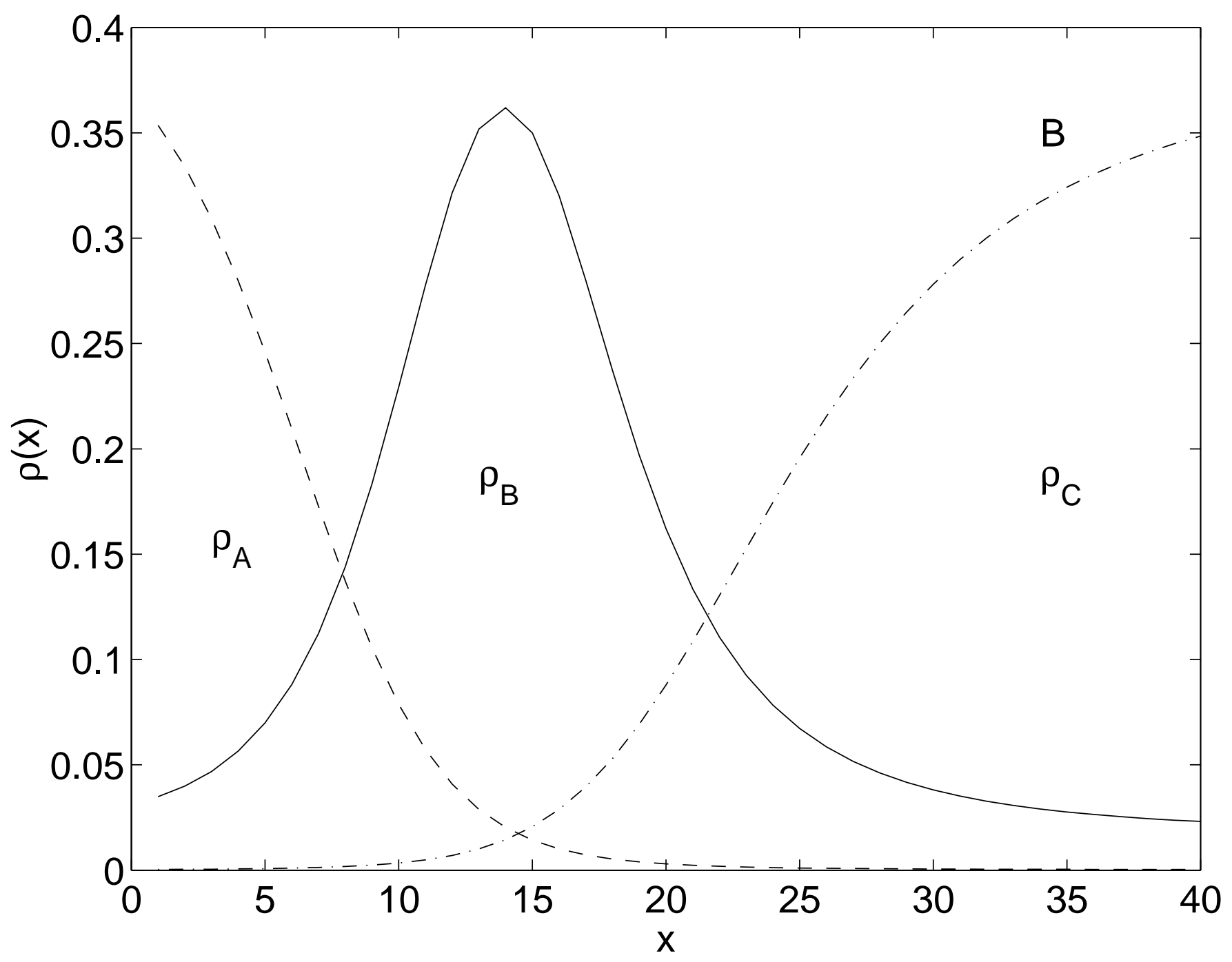

Figure 6B 


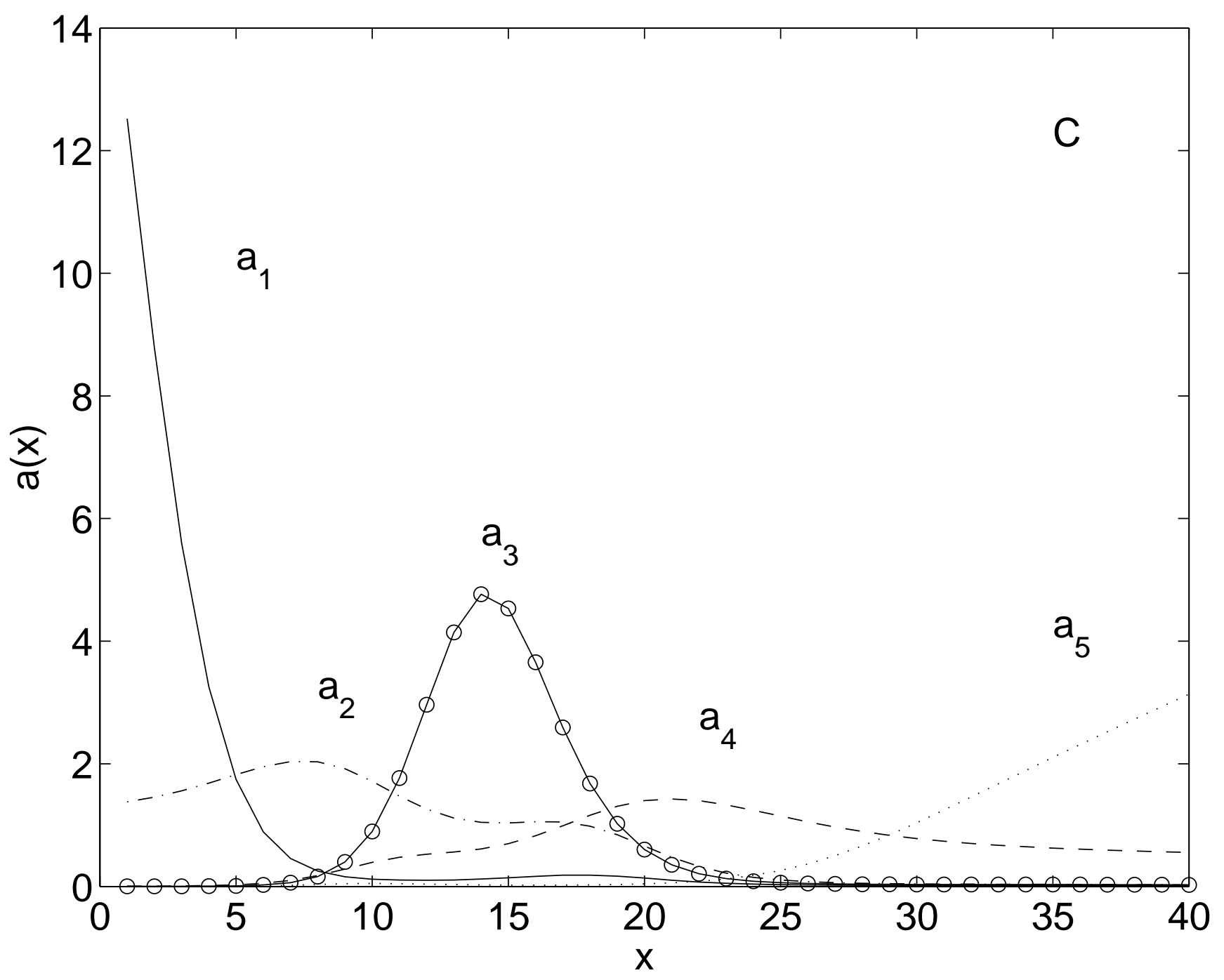

Figure 6C 


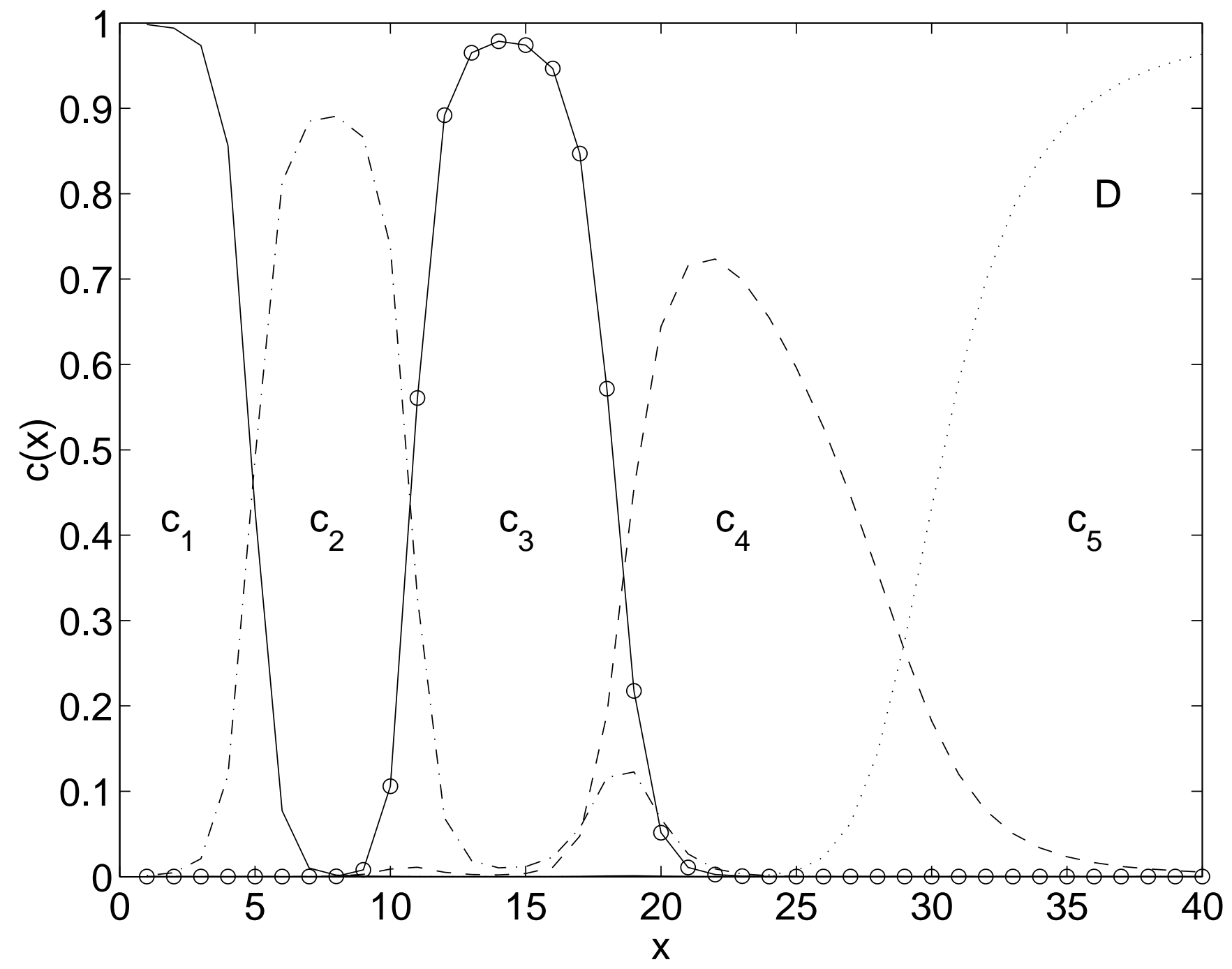

Figure 6D 


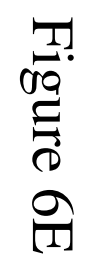

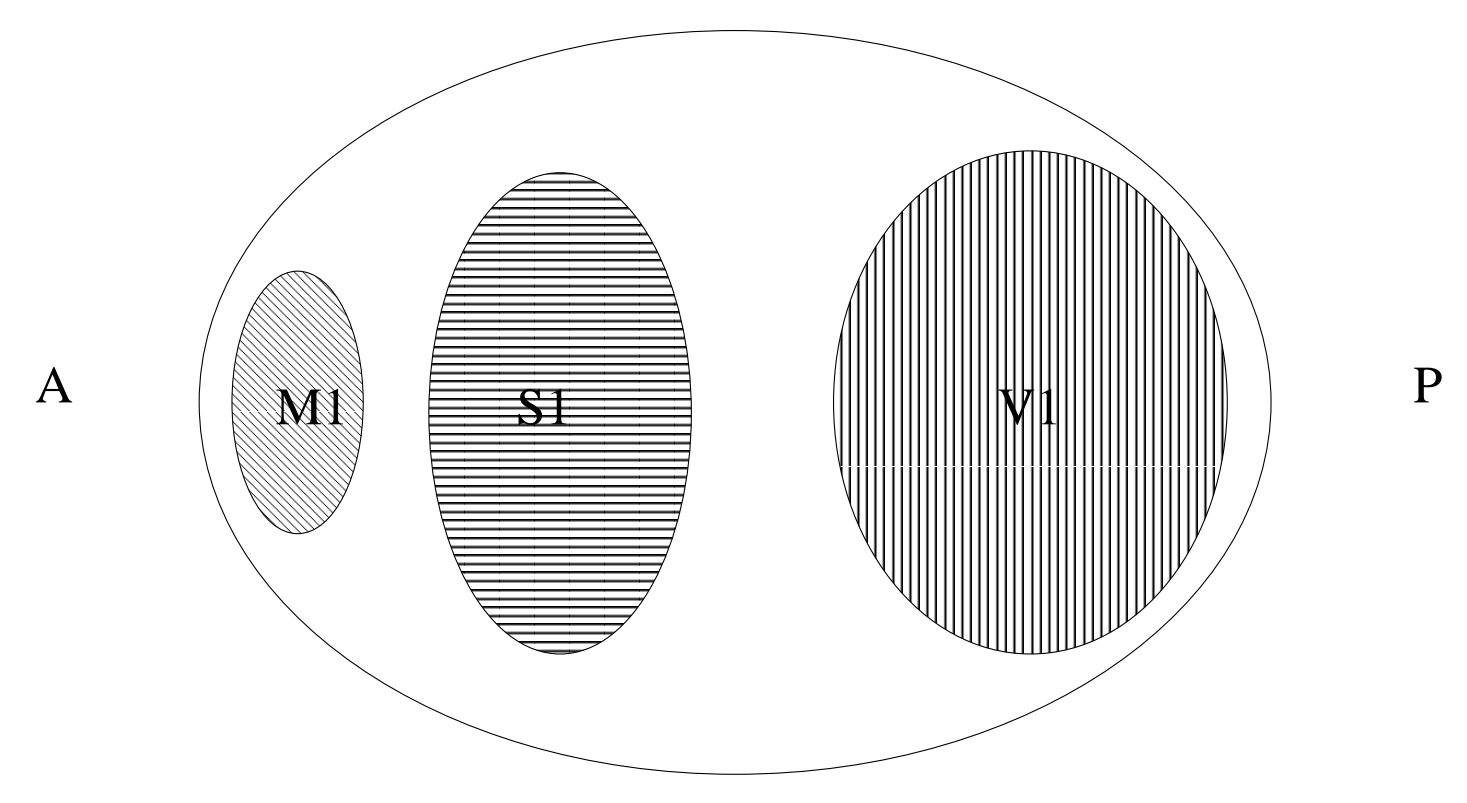




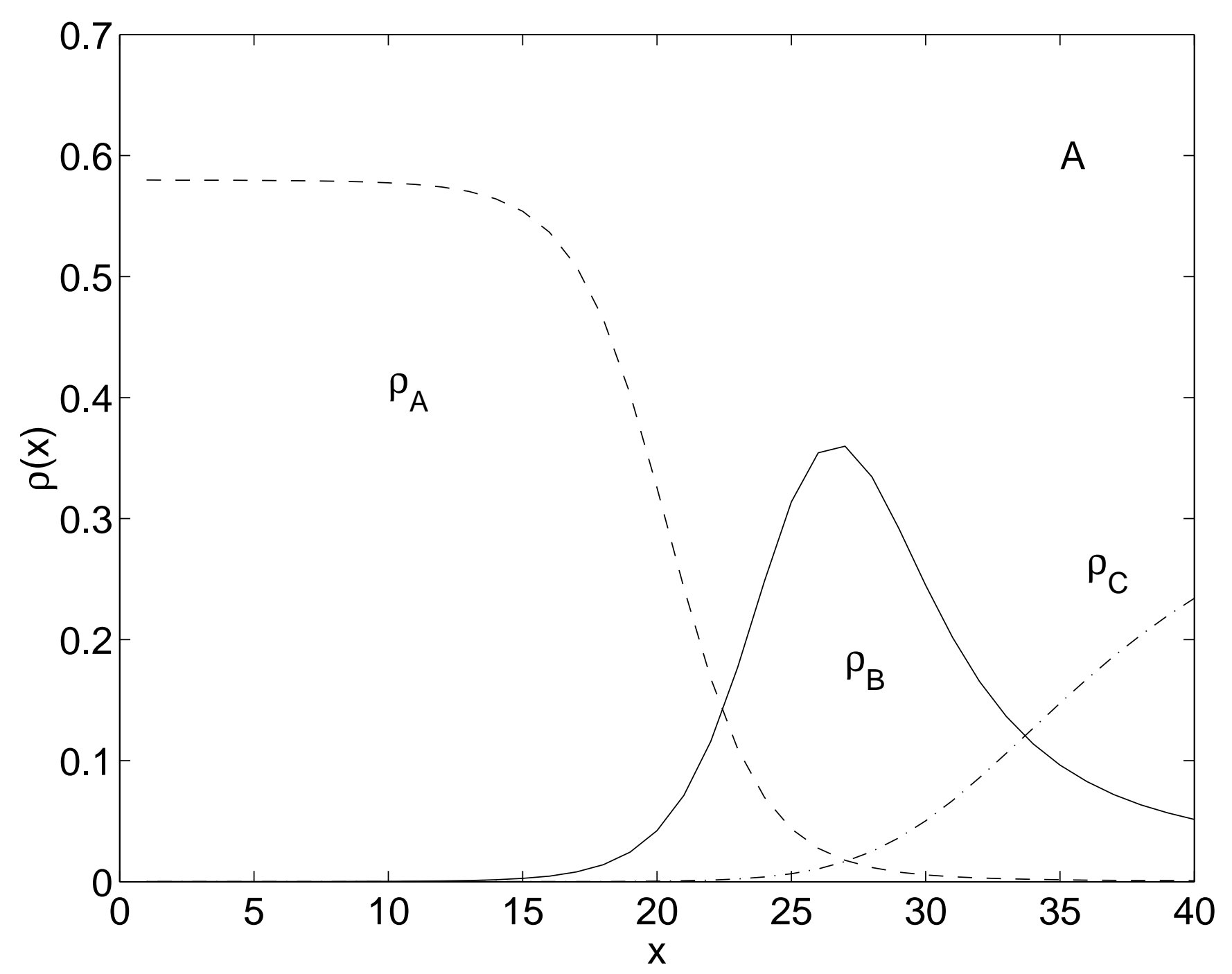

Figure 7A 


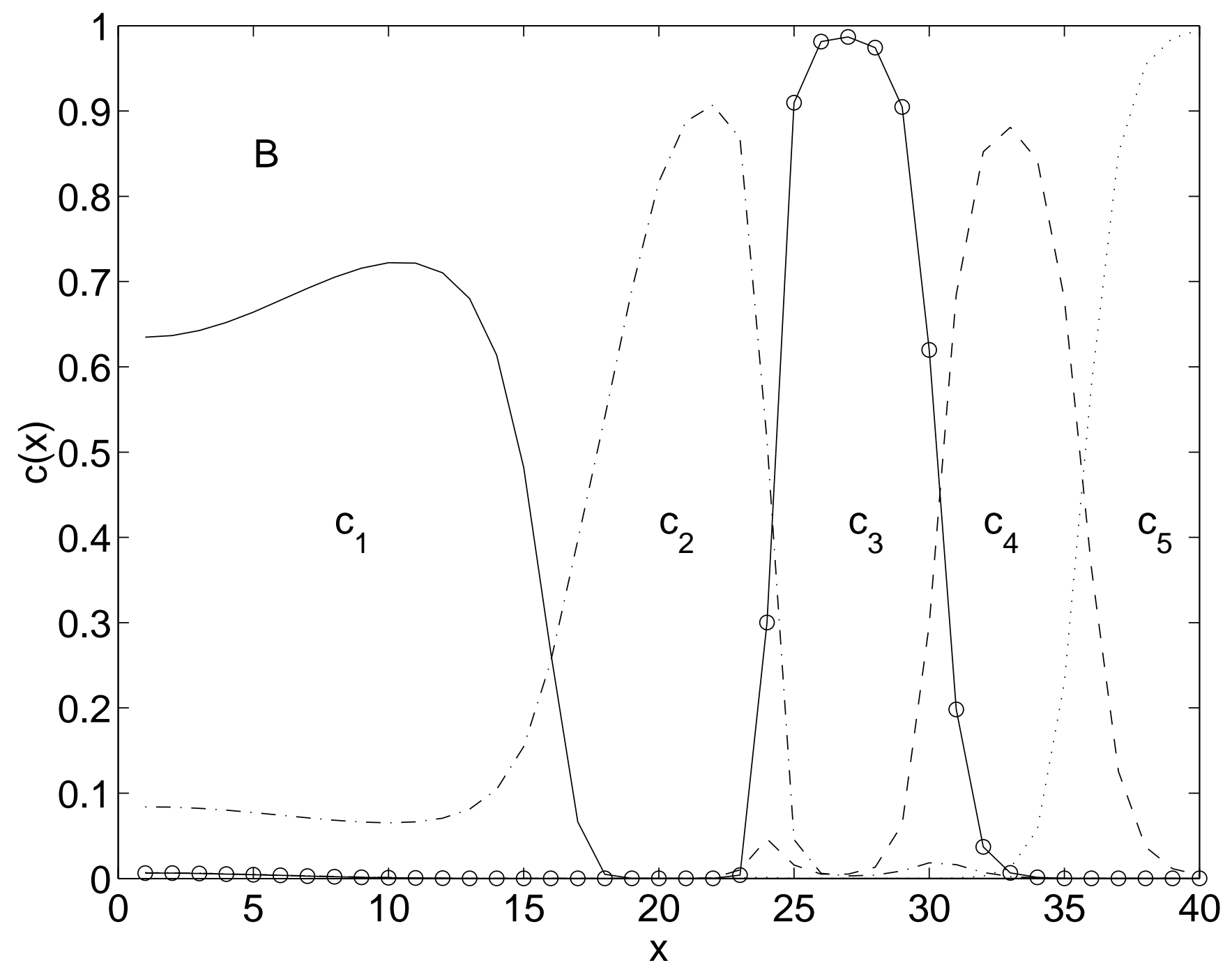

Figure 7B 
กับ

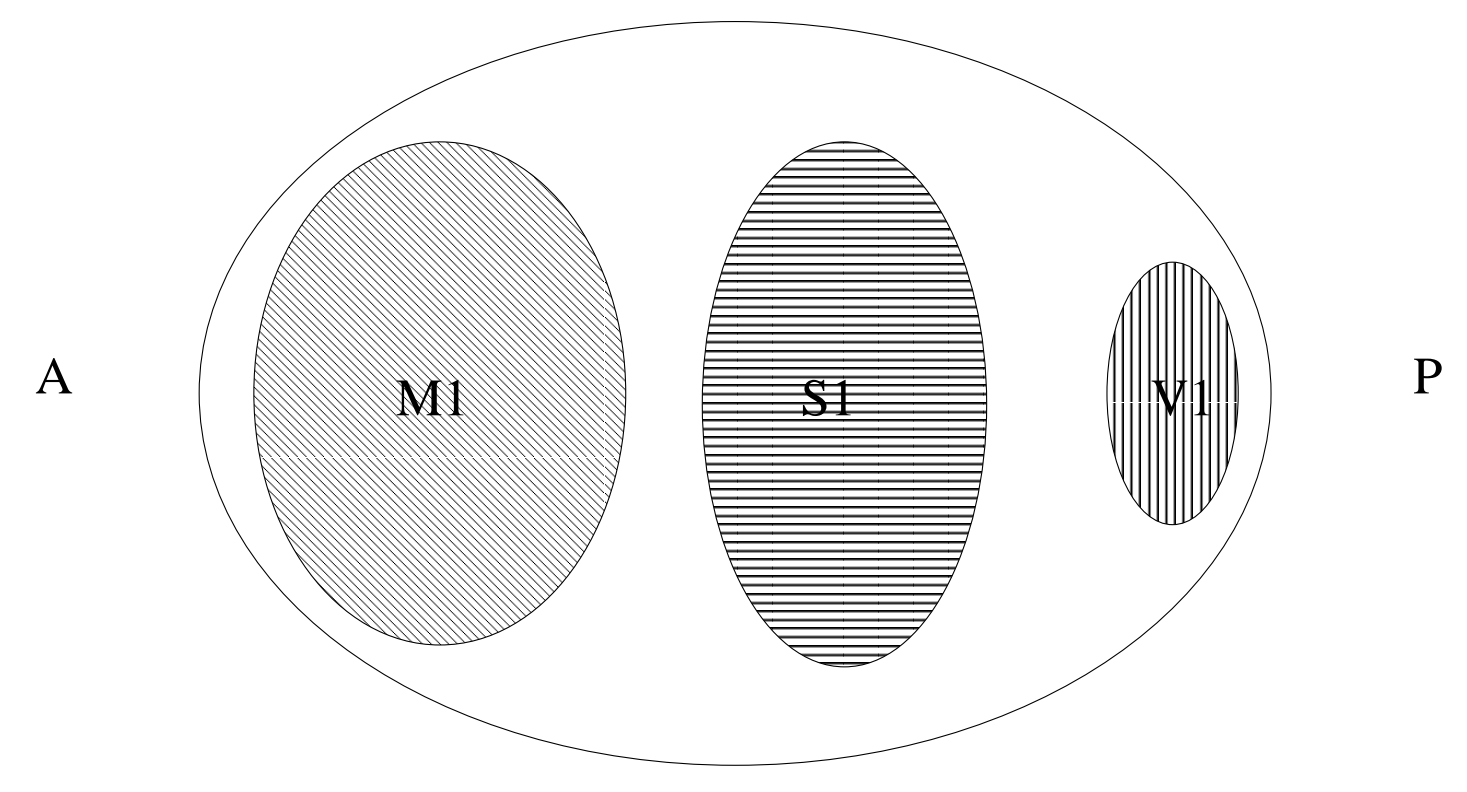




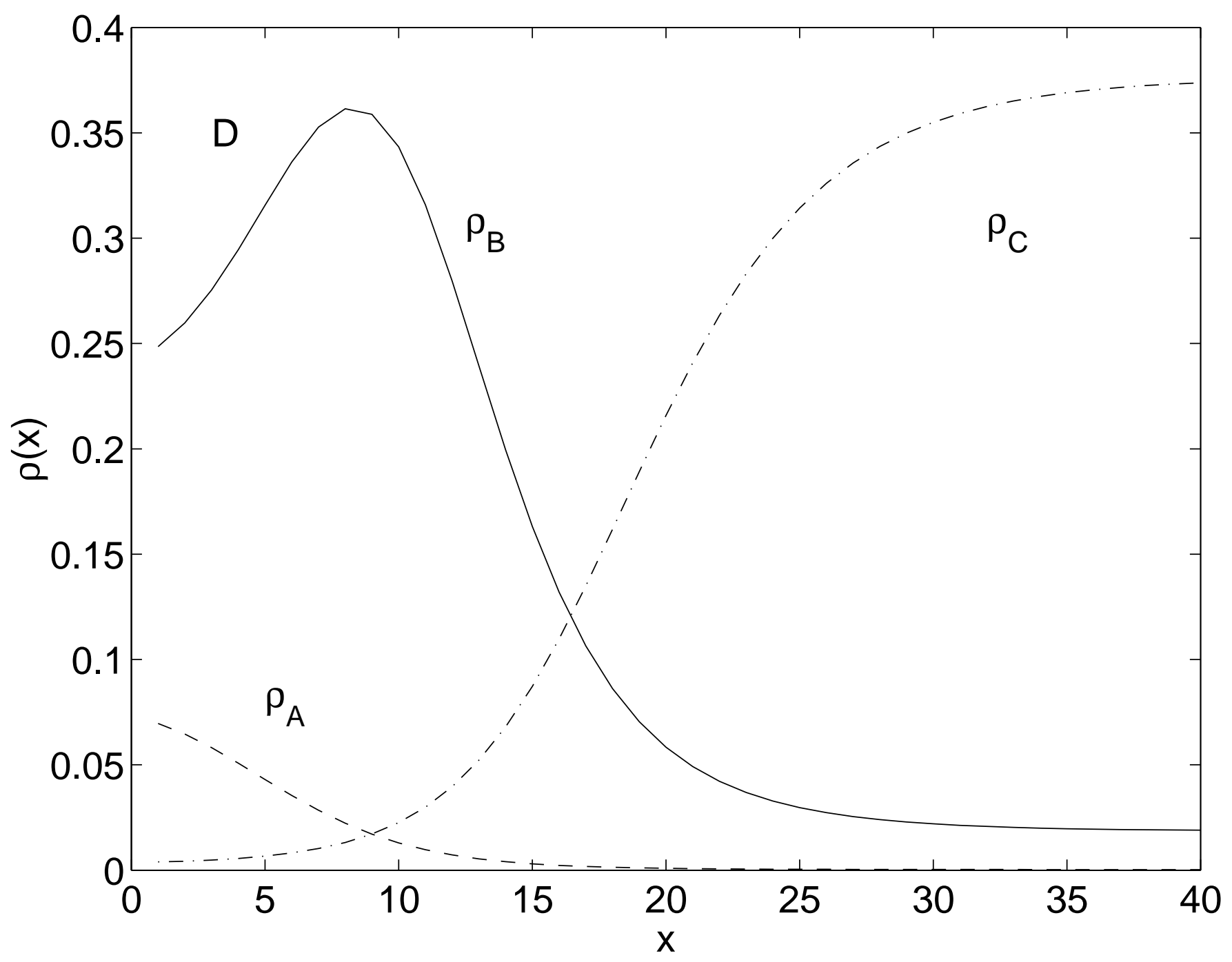

Figure 7D 


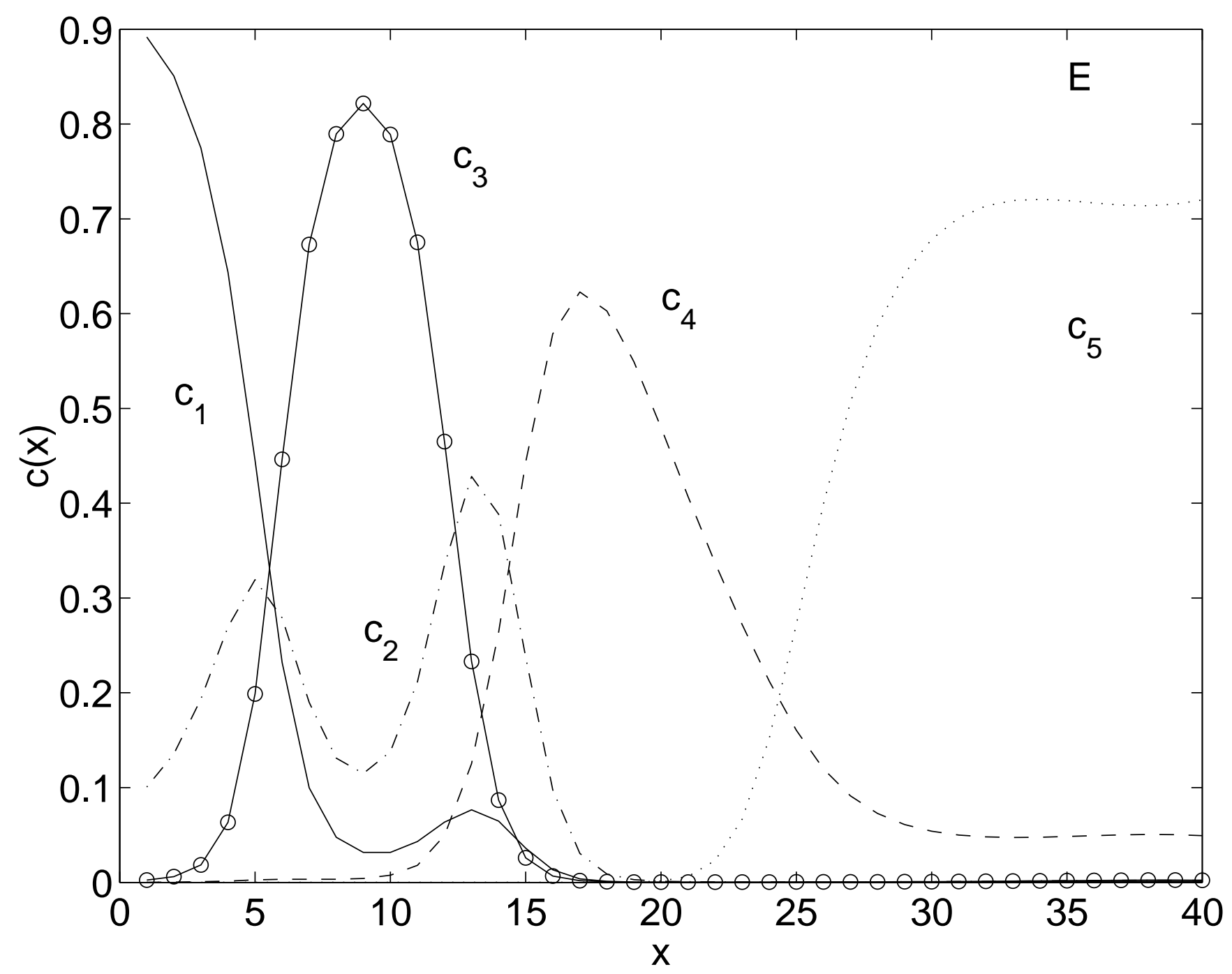

Figure 7E 
章

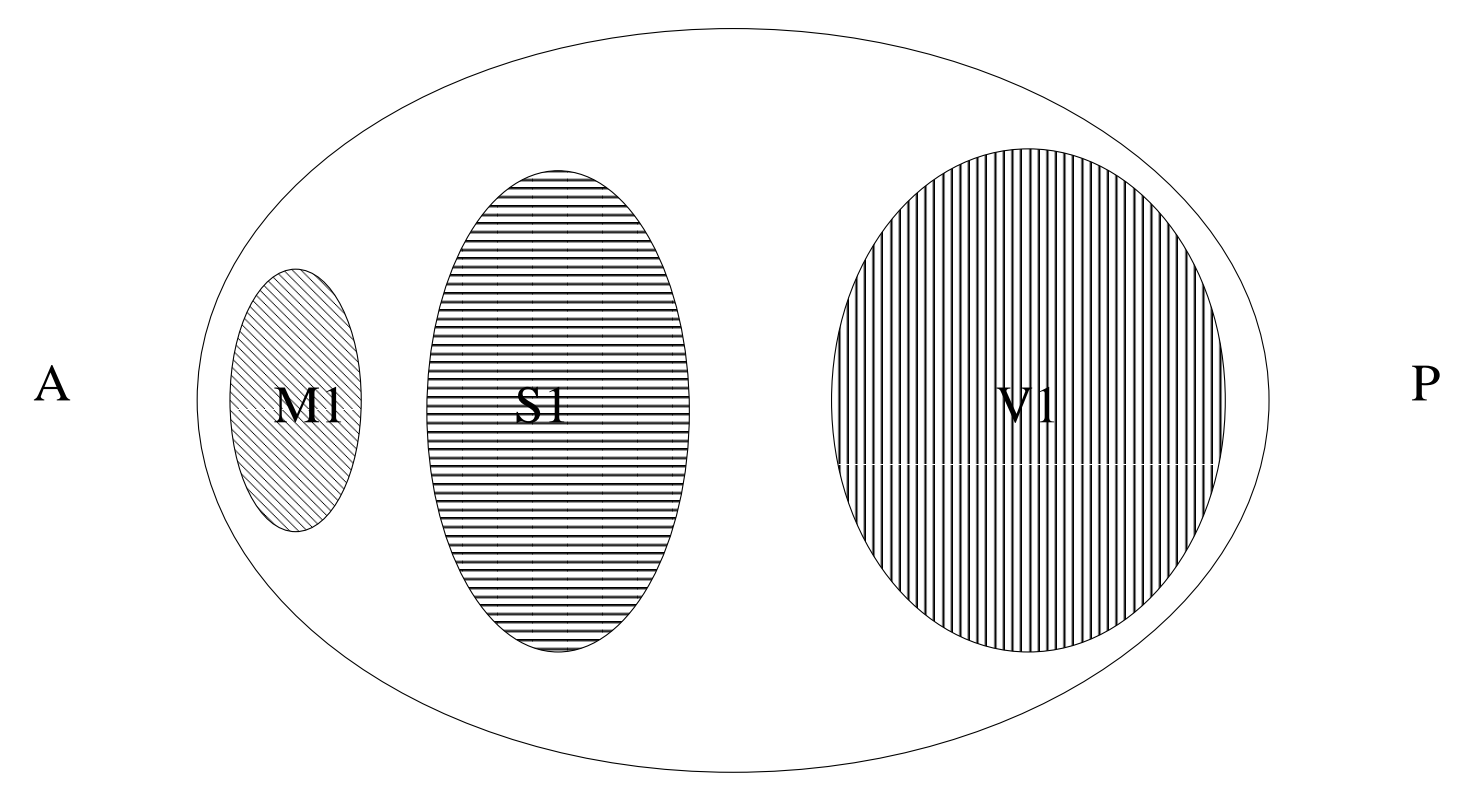




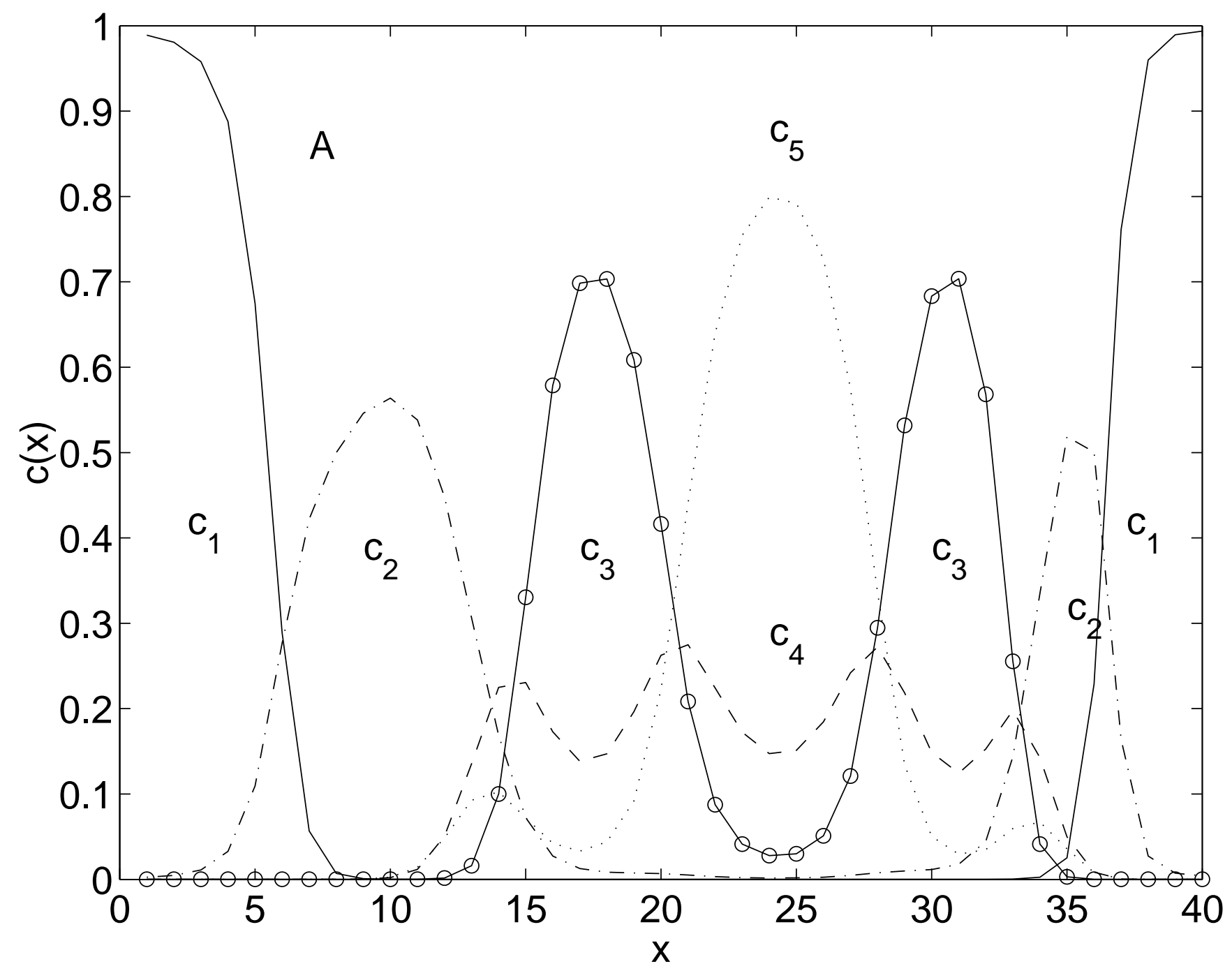

Figure 8A 

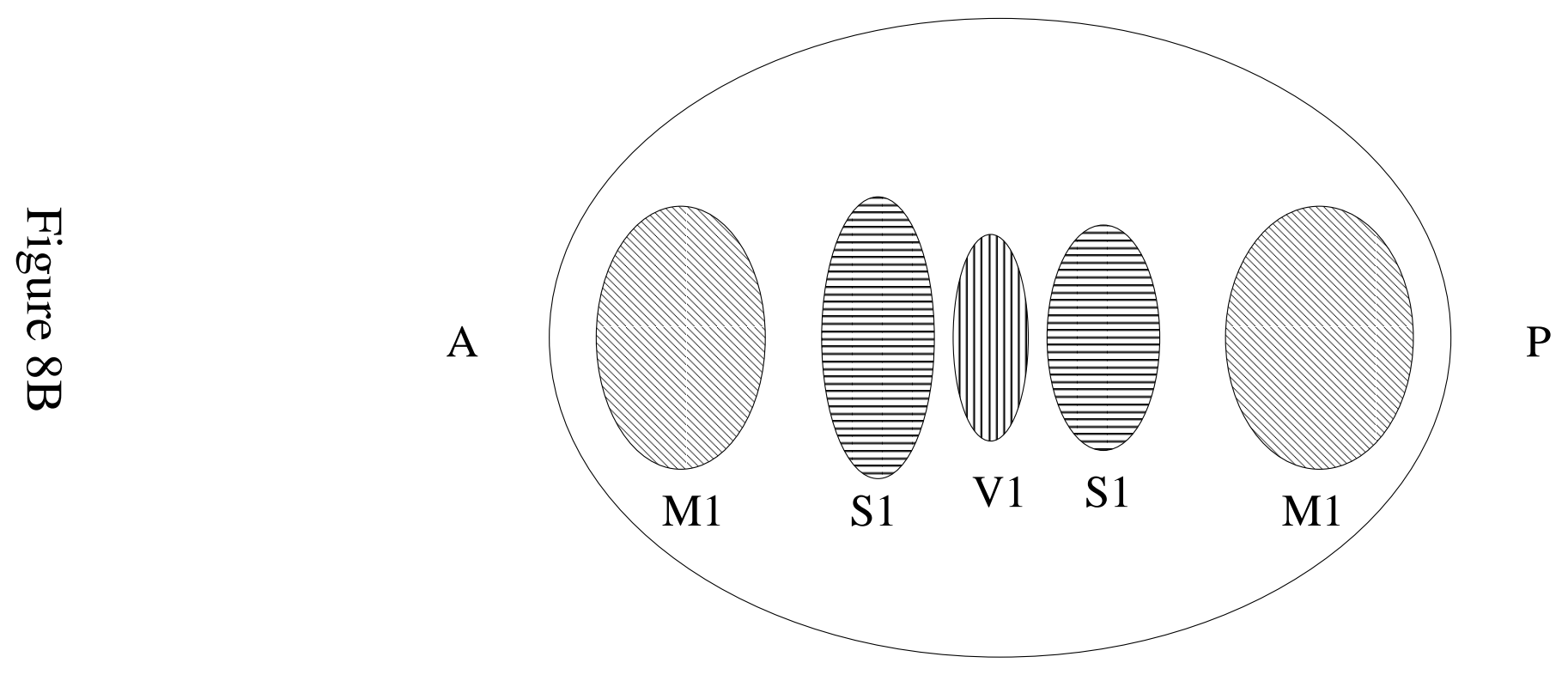


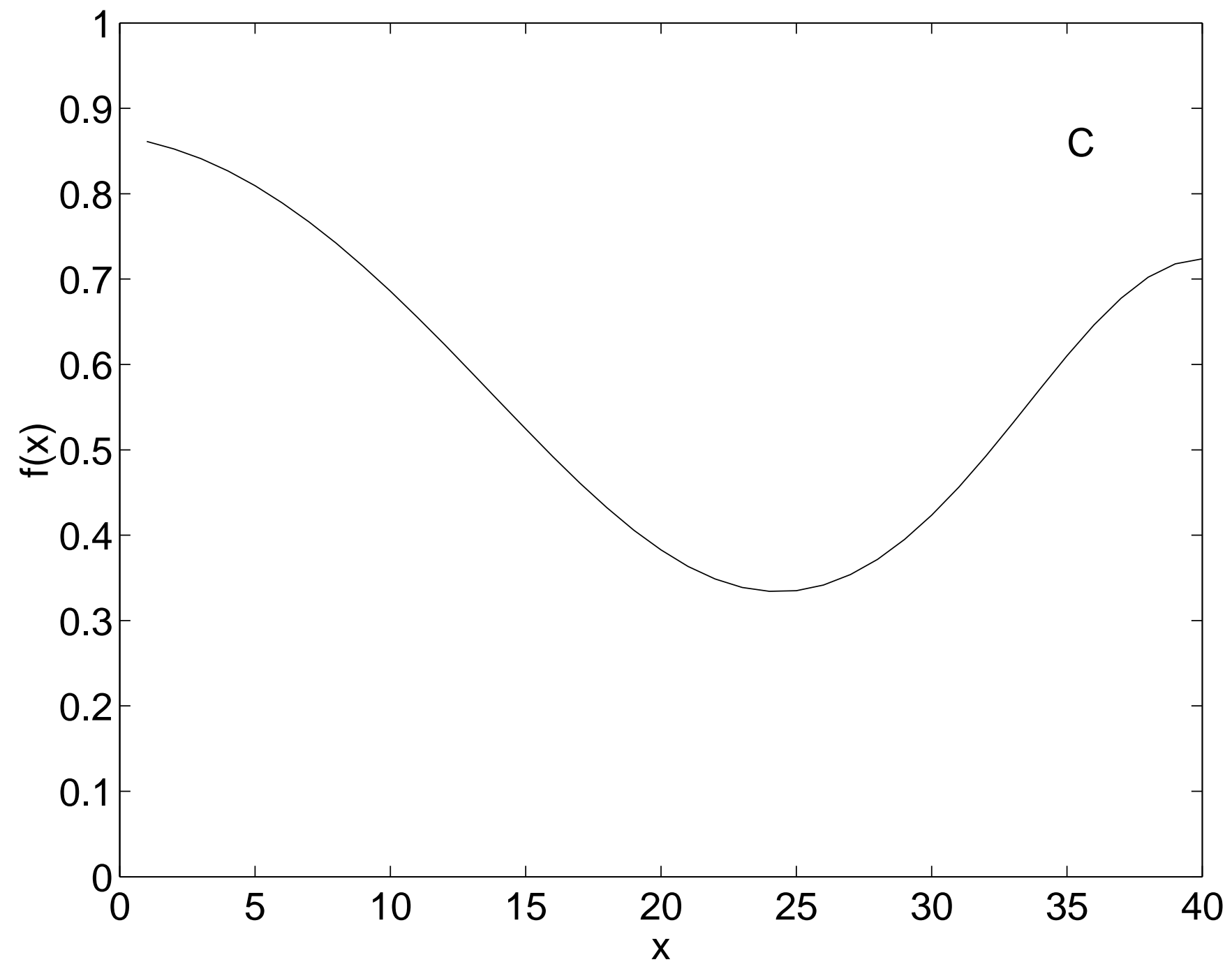

Figure 8C 


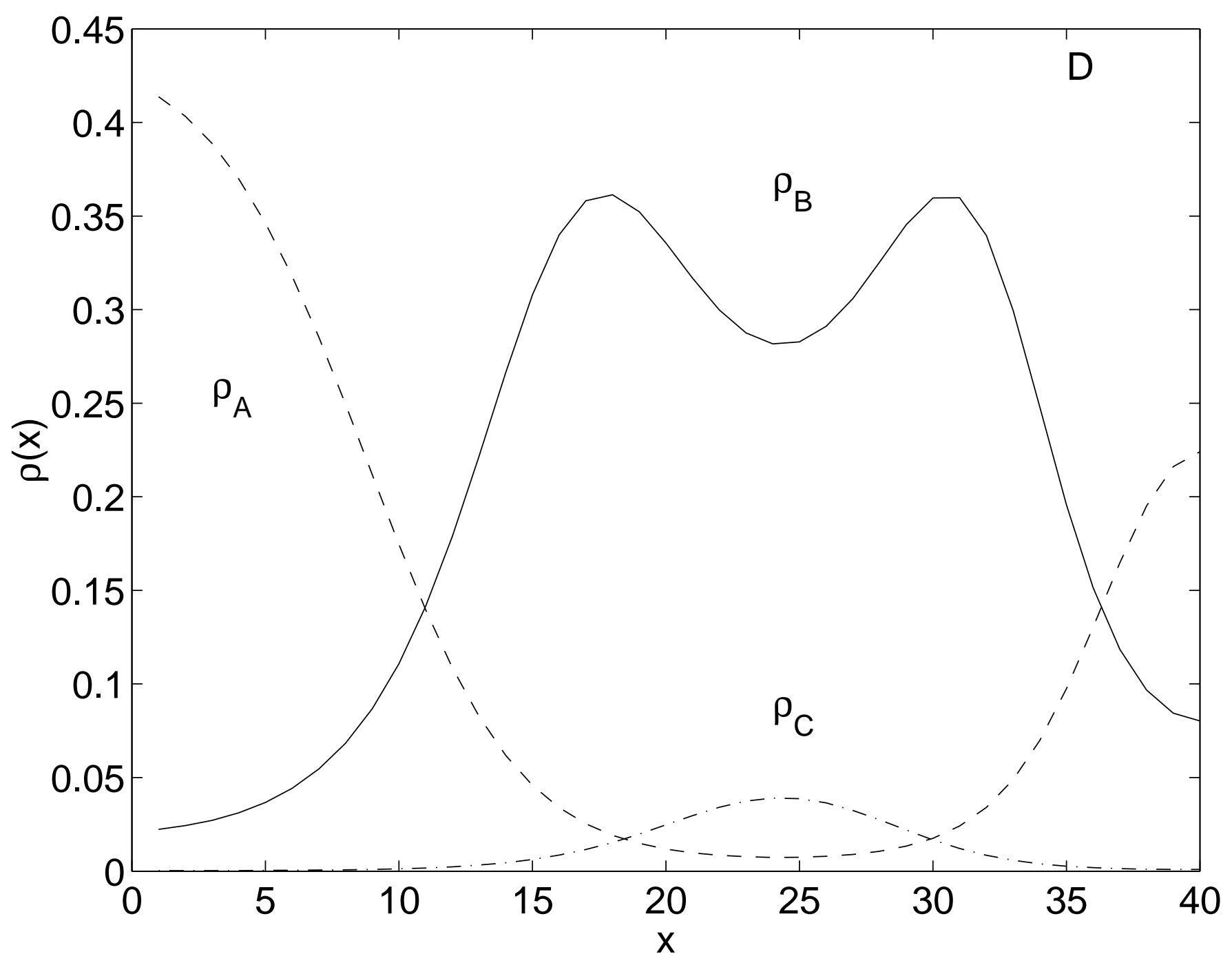

Figure 8D 


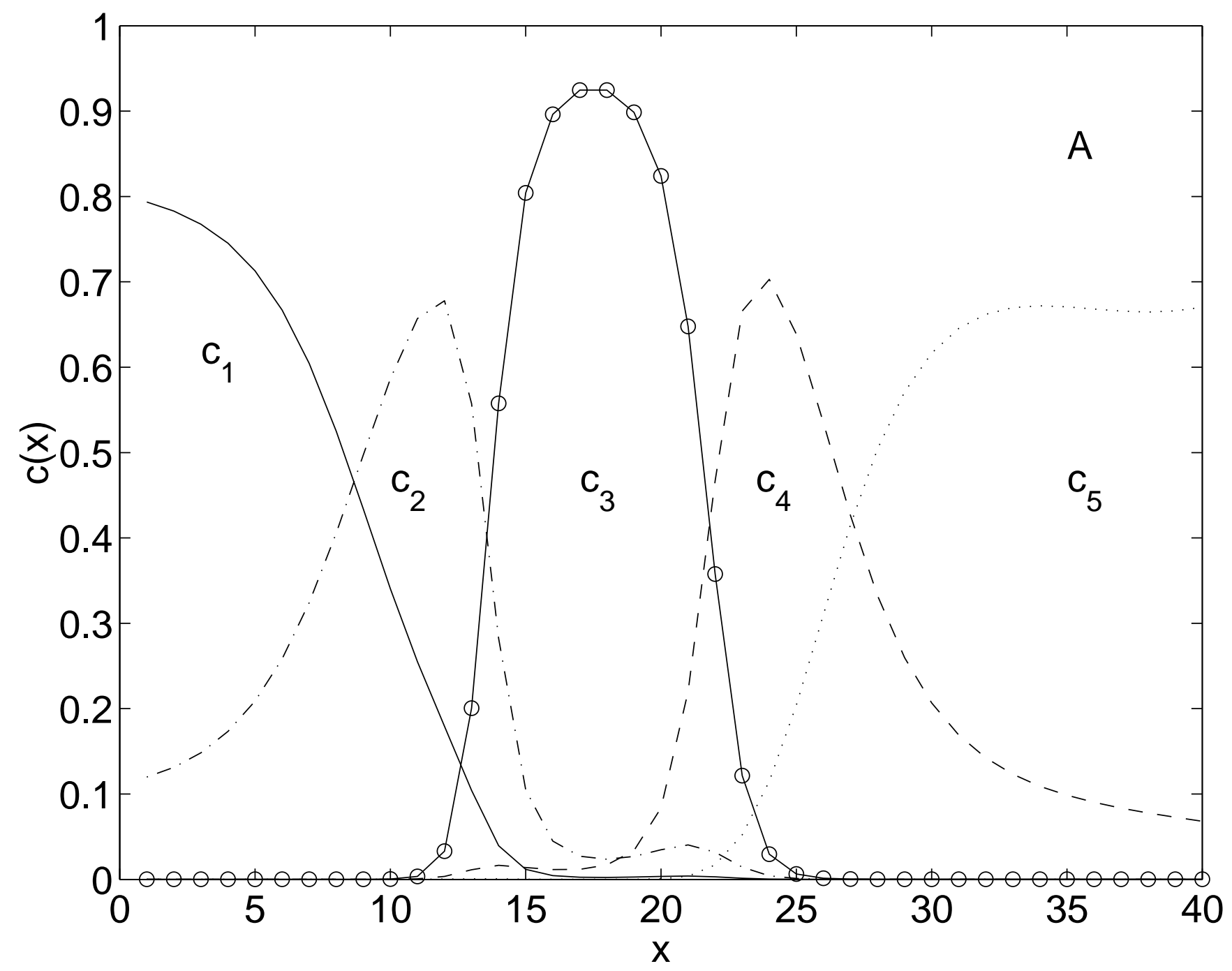

Figure 9A 


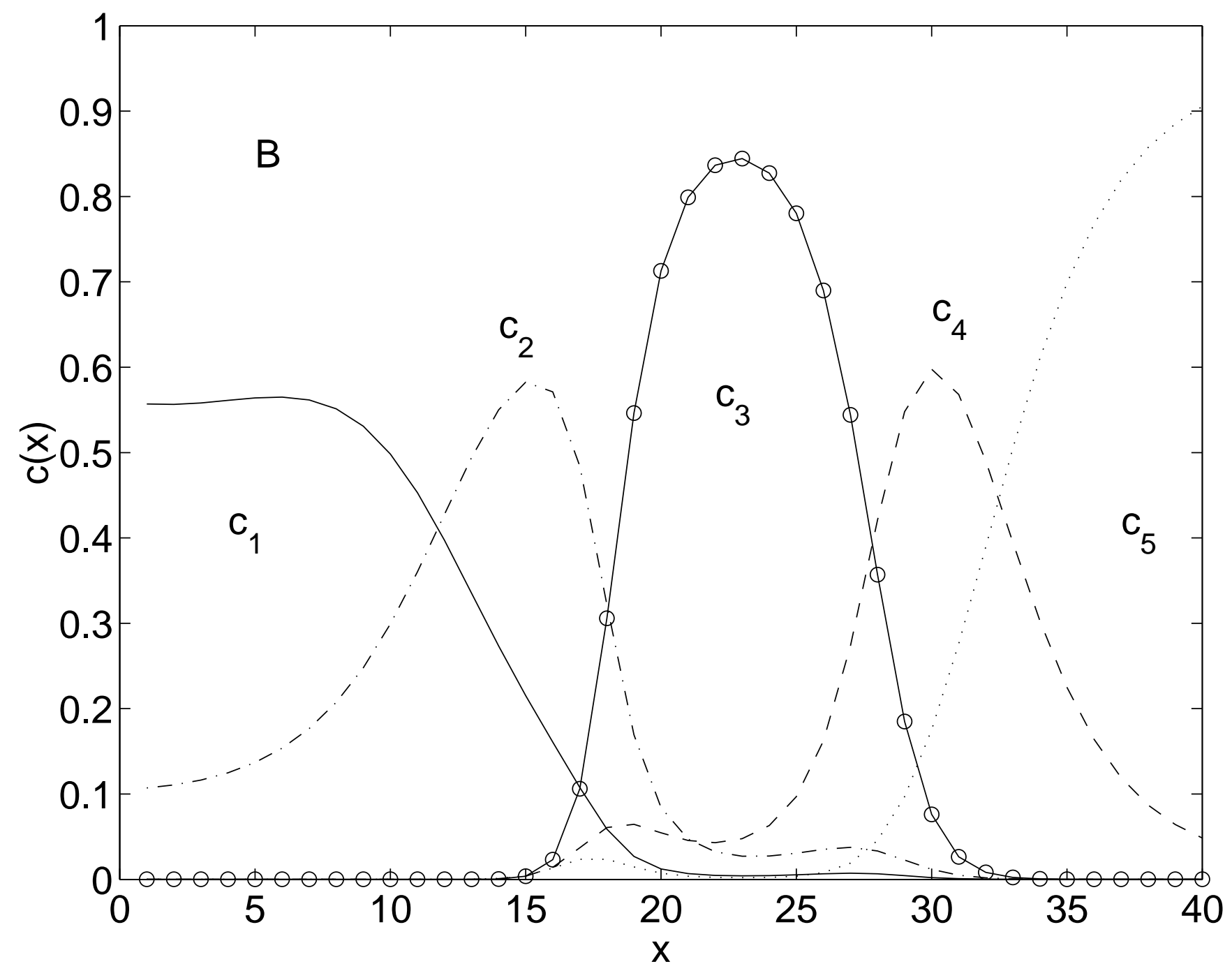

Figure 9B 


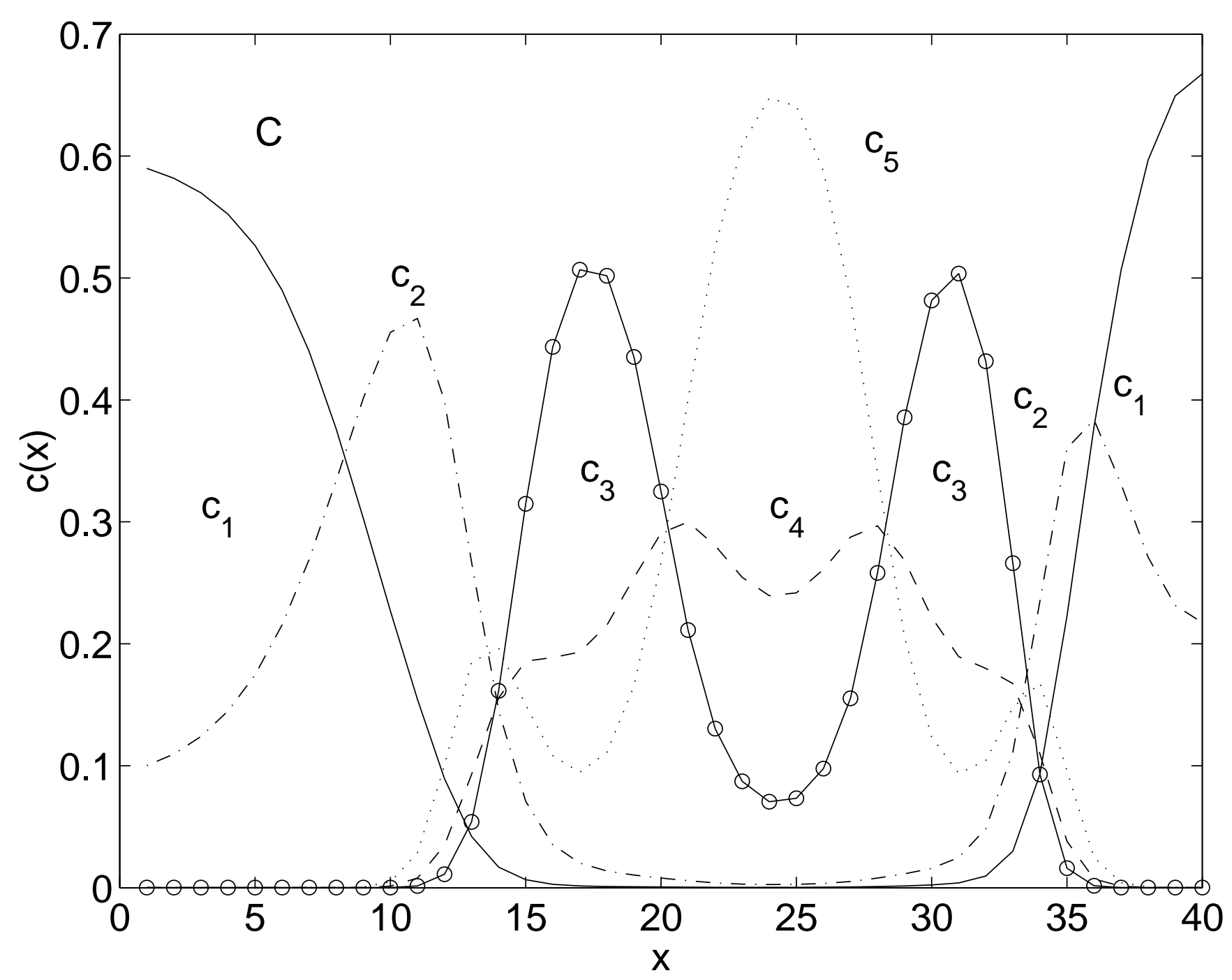

Figure 9C 